\title{
Book reviews - Comptes rendus - Buchbesprechungen
}

\author{
Roland DANNREUTHER (ed.) - European Union Foreign and Security Policy. \\ Towards a neighbourhood Strategy, Routledge, London and New York, 2004, 225 \\ p. - ISBN 0-415-32298-7 - 26,68 €.
}

Le débat actuel qui consiste à savoir si l'UE est un acteur international alimente de plus en plus la production éditoriale, signe indéniable de l'intérêt porté à ce questionnement, dont les conséquences sur le thème de l'identité européenne sont fortes. Il est abordé dans ce livre, édité par Roland Dannreuther, suivant une perspective particulière, qui consiste à juger si cette même UE défend une vision cohérente dans son environnement immédiat. C'est ainsi que sont passés en revue les différents pays et régions où l'Europe peut, ou pourrait développer une politique étrangère et de sécurité communes: Europe centrale et orientale bien entendu (Pál Dunay), Turquie (Gilles Dorronsoro), Europe sud-orientale (Ettore Greco), Russie, Ukraine, Moldavie et Belarus (Andrei Zagorski), Scandinavie (Hiski Haukkala), Caucase et Asie centrale (Neil MacFarlane), Afrique du Nord (Fred Tanner) et Moyen Orient (Roland Dannreuther), études empiriques auxquelles il faut ajouter des approches plus thématiques, celles d'Antonio Missiroli sur la notion de «voisinage», de William Wohlforth sur le lien transatlantique, et de John Gault sur la sécurité énergétique.

Onze cas au total sont étudiés, donnant un éclairage sur le rôle de l'UE dans certaines politiques de voisinage, avec une attention particulière au processus d'élargissement en Europe centrale et orientale, à l'engagement dans les Balkans, au conflit israélo-arabe, et à la politique à l'égard des pays de l'ex-URSS. Chaque contribution répond à cinq thèmes de recherche: le développement de politiques communautaires pertinentes (ou non) depuis la fin de la Guerre froide; l'impact de l'influence stratégique de l'UE; l'étendue de l'autonomie de cette dernière par rapport aux politiques des Etats membres; l'influence d'acteurs extérieurs dans les affaires européennes; le passage ou non de l'UE d'un statut de puissance civile à celui d'acteur stratégique. Le livre a paru dans les premiers mois de l'année 2004, avant donc l'adhésion officielle des dix à l'UE, et ne tient pas compte de certains événements forts ayant trait aux thèmes étudiés, tels que la révolution orange en Ukraine ou l'ouverture des négociations d'adhésion avec la Turquie.

Dans l'introduction, Roland Dannreuther, professeur à l'université d'Edimbourg, lui-même spécialiste des relations internationales, rappelle que la politique européenne de voisinage est une conséquence directe de l'après-Guerre froide: la nécessité de s'engager dans la stabilisation des pays de l'ancien Pacte de Varsovie s'est imposée peu après l'écroulement de l'hégémonie soviétique après 1989, et le résultat de ce fait fut et reste un défi stratégique et géopolitique consistant à remplir un vide inquiétant. Qui plus est, la logique interne de l'intégration européenne stipule que l'acquis communautaire ne saurait être limité à l'ouest mais devrait être étendu à l'est; or, l'avenir du projet d'intégration est lié au succès de l'engagement 
stratégique de l'UE dans son environnement immédiat. Selon Dannreuther, ce défi a trois dimensions: l'élargissement, la Politique étrangère et de sécurité commune (PESC), et la Politique européenne de sécurité et de défense (PESD), tous trois devant faire face aux divers défis sécuritaires issus de la périphérie de l'UE. Le but est donc de juger jusqu'à quel point l'Europe occidentale, UE et Etats membres, a été capable de se comporter de manière cohérente à l'égard des pays voisins depuis la fin de la Guerre froide.

Afin de savoir si les différentes politiques et stratégies en direction du voisinage immédiat font apparaitre l'UE comme un acteur stratégique cohérent, les contributeurs de ce volume suivent une approche empirique et analytique, ce qui fait que le titre peut être considéré comme trompeur: l'aspect procédural et les mécanismes institutionnels propres à une diplomatie «européenne» ne sont pas traités dans cet ouvrage, alors que les sources principales de l'action et des programmes (Commission et Conseil) ne sont pas vraiment abordées. Le but central de l'ouvrage est de démontrer qu'une politique étrangère européenne émerge par-delà les politiques nationales de coordination, les politiques supranationales et celles qui sont basées sur la formule intergouvernementale de la PESC. Les différents systèmes sont considérés comme étant solidement interconnectés, dans le même temps où les auteurs reconnaissent que la coopération pâtit d'un certain manque de coordination. Cela dit, l'influence de l'Europe apparait plus forte qu'une simple analyse de la seule PESC peut le suggérer, ce qui souligne la complexité du processus décisionnel de l'UE en la matière.

Même s'il est ainsi difficile d'obtenir une photographie complète de la mosaïque des polices et stratégies de l'UE, l'ouvrage cherche à fournir une conceptualisation cohérente des politiques étrangères de l'UE en direction des régions voisines et à voir si l'UE peut être considérée comme un acteur stratégique par celles-ci. L'originalité est d'utiliser une perspective stratégique et de contribuer dans le même temps aux débats plus larges à propos de l'UE comme acteur international. Cette approche est suivie par tous les contributeurs, qui essaient de répondre au formulaire de recherche décrit ci-avant, en analysant les différentes politiques et stratégies de l'UE.

A la suite de ces différentes analyses, Roland Dannreuther tire la conclusion que «l'ambiguiité en apparence insoluble de son identité externe» reste la principale faiblesse de l'UE. Qui plus est, l'ouvrage ne tient pas compte de l'élargissement de l'UE à 25 membres, qui a d'ores et déjà compliqué une carte stratégique de l'Europe déjà peu claire. Les auteurs reconnaissent de fait l'apparition d'une certaine «fatigue de l'élargissement» chez les Occidentaux, alors que la perspective de l'adhésion est vue par les candidats comme une simple «carotte économique», bien que l'élargissement ait pour finalité le but fortement politique et stratégique de promouvoir la stabilité dans le voisinage. La question de la géométrie variable est ainsi reposée en fin d'ouvrage, afin de repenser un système qui doit tenir compte de la délicate question de la souveraineté dans un ensemble à 25, tout en s'interrogeant sur l'attitude à tenir vis-à-vis de la nouvelle périphérie d'une UE 
fortement excentrée vers l'Est. Ainsi, toutes les contributions mettent en avant le fait que le processus décisionnel de l'Europe, trop opaque et bureaucratique, rend une ligne d'action cohérente de plus en plus difficile à obtenir. La principale raison à cela est le caractère sui generis de l'UE. Celle-ci n'est pas un acteur unitaire avec une autorité centrale en matière de politique étrangère et de sécurité, ce qui mène à ce problème général, dont pâtit toute velléité d'action commune: les politiques de l'UE sont souvent riches en rhétorique, alors qu'elles manquent des ressources appropriées et des mécanismes pour atteindre les buts désignés, le principe de la conditionnalité par exemple n'étant pas forcément le garant d'une véritable réussite en matière de démocratie s'il n'est pas accompagné de demandes contraignantes. Les politiques européennes restent trop souvent conduites par des facteurs externes, et gênées par la logique complexe du processus de décision interne de l'UE, ce qui amène à différentes formules provisoires ou des politiques non coordonnées à l'égard d'un «extérieur» que tous les acteurs ne voient pas de la même manière. Or, la PESC a une importance capitale concernant l'identité européenne: interne (penser à la finalité géographique) comme externe (statut et rôle dans l'ordre international).

Ainsi, ce livre présente un travail fondé et cohérent en analysant l'engagement stratégique de l'UE dans sa périphérie, audaces et résistances comprises. Même s'il y a des différences en termes de qualité concernant les articles, toutes les contributions fournissent une analyse convaincante et bien écrite. Certes, l'approche d'ensemble du livre est très descriptive, et cela aurait été plus intéressant encore si les auteurs avaient engagé une discussion un peu plus théorique dans leurs analyses (ce qui est parfois le cas, comme dans la contribution de William Wohlforth).

Ce livre va sûrement susciter l'intérêt de toute une série de lecteurs. Ces derniers trouveront une riche analyse empirique des différentes régions qui «voisinent» l'Europe, ou pourront s'intéresser à l'impact de l'UE dans certains secteurs clés, ainsi qu'à une politique étrangère commune pour l'instant éclatée, parfois plus contingente qu'intentionnelle.

Bertrand Vayssière Maître de conférences à l'université de Toulouse II-Le Mirail

Roland DANNREUTHER, John PETERSON (eds) - Security Strategy and Transatlantic Relations, Routledge, London and New York, 2006, 262 p. - ISBN 0-415-40189-0 - 30,03€.

Issu d'un double colloque de l'UACES (University Association for Contemporary European Studies, réseau de différentes universités européennes), cet ouvrage rassemble les contributions d'auteurs d'horizons divers: Ronald D. Asmus (German Marshall Fund), Alyson Bailes (Stockholm International Peace Research Institute), Fraser Cameron (European Policy Centre), Anoushivaran Ehteshami (Durham), Jolyon Howorth (Yale), Annika Bergman, Luker March et Chad Damro 
(Edimbourg), Sean Molly (Glasgow) et Jim Wyllie (Aberdeen). Le livre peut lui-même être décrit comme le premier traitant de l'impact du National Security Strategy (NSS) américain (2002) et de la Stratégie européenne de sécurité (SES) de l'UE (2003) sur les relations transatlantiques. Ces nouvelles stratégies sont analysées comme tentatives de reconceptualiser la nature radicalement changeante de la sécurité internationale après le 11 septembre, et de redéfinir des doctrines adaptées à l'après-Guerre froide. Etudier celles-ci permet de percevoir les tensions transatlantiques, particulièrement pendant et après la guerre en Irak, tout en observant les points communs et les différences entre deux regards sur le monde.

Cet objectif a été atteint par un groupe de travail de l'UACES, qui s'est d'abord réuni à Edimbourg, en février 2005, puis à Bruxelles en septembre. Tous les contributeurs ont dû répondre à trois questions clés, en relation avec leurs sujets d'analyse: jusqu'à quel point les stratégies de sécurité américaine et européenne convergent ou divergent-elles? Quelles politiques tangibles peuvent être vues comme le résultat de ces nouvelles stratégies, particulièrement dans le secteur de la «guerre contre le terrorisme»? Quelles sont les implications des stratégies de sécurité américaine et européenne à l'égard de certaines régions spécifiques, surtout le Moyen Orient, la Russie et la Chine?

Voici réunies les principales conclusions de l'ouvrage:

Toutes les contributions considèrent que les NSS et SES ne sont pas en soi des documents stratégiques de premier ordre, mais de simples documents de travail. $\mathrm{Ce}$ sont donc avant tout des aspirations à être à la hauteur des défis lancés par un environnement international qui a incontestablement changé. Ces documents n'affectent cependant pas l'autonomie stratégique des décideurs des deux côtés: les deux stratégies de sécurité restent d'ailleurs différentes, même si toutes deux expriment des ambitions nouvelles. Les Américains essaient de renouer avec un passé où politique étrangère et stratégique et «destinée manifeste» sont étroitement liées; la SES, de son côté, a une ambition différente, première tentative de donner un sens concret à ce qu'un acteur international européen serait et quelle valeurs il pourrait défendre: inévitablement, cette tentative entraîne une certaine forme d'autonomisation vis-à-vis des Etats-Unis et de l'OTAN, à la base d'une identité européenne. En ce qui concerne les convergences et les divergences, l'ouvrage ne confirme pas la conception populaire que le fossé s'élargirait entre Europe et Amérique: un consensus général émerge même plutôt autour de l'idée que les deux ensembles convergeraient dans l'ambition clé d'une «diplomatie de transformation». Etats-Unis et Union européenne sont également présentés comme des modèles de stabilité, de liberté et de prospérité, ce qui renforce leur volonté de changer le monde à leur image. Il semble également y avoir convergence dans l'identification des principales menaces aux valeurs communes, ressenties comme telles par les deux parties prenantes. Autant le NSS que la SES identifient le terrorisme, la prolifération des armes de destruction massive, les conflits régionaux, et les Etats faibles comme des défis réclamant une solution urgente. Certes, le NSS use d'un ton très sceptique, alors que la SES se montre plus optimiste dans son évaluation des bénéfices de l'après-Guerre froide. Mais, en substance, les deux 
documents se rejoignent pour dessiner le même monde extérieur et fournir la même stratégie de base.

Les divergences concernant les moyens et les instruments pour atteindre ces mêmes buts existent parallèlement: les Européens ont été particulièrement choqués par le ton jugé «néo-impérialiste» du NSS, ainsi que son triomphalisme; pour beaucoup d'Américains, la SES n'est pas satisfaisante, et signifie un échec de l'Europe, incapable d'aider une Amérique blessée. Le NSS se révèle être un document relativement réaliste, à la recherche de cette balance of powers, seule capable de favoriser la paix (issue signalée cinq fois dans le document) grâce à l'utilisation des moyens adéquats pour faire respecter et triompher une approche d'inspiration wilsonienne pour l'expansion de la liberté. Ainsi, le document appuie une prétention universaliste d'intégration socio-économique. Le modèle européen, lui, croit plus dans la persuasion que dans la coercition. Le temps peut cependant gommer les différences: le besoin de stabiliser l'Irak a fini par rassembler les points de vue, même évolution pour la Corée du Nord ou l'Iran concernant les armes de destruction massive.

L'avenir de ces deux documents est présenté de manière très prudente, voire négative. Les contributeurs sont d'accord pour démontrer les échecs des stratégies de sécurité américaine comme européenne dans de nombreux secteurs (Russie de Poutine, Chine, lutte contre Al Qaeda). De manière plus particulière, l'Irak a représenté beaucoup pour le NSS, mais l'échec semble patent: la rhétorique de liberté s'est concrètement transformée en renforcement de la domination chiite dans le pays, expansion de l'influence iranienne et peur croissante des régimes sunnites du Golfe à l'extérieur. Tout cela pourrait bloquer la transformation voulue de la région. De fait, l'avenir du NSS est largement lié aux évolutions ultérieures sur le terrain, et explique l'intransigeance de Washington en même temps que ses attentes. Pour la SES, le problème est différent, et repose d'abord sur sa nature et sa nouveauté: est-ce seulement une déclaration d'intention, des vœux pieux? C'est vrai que le NSS s'est frotté à l'Irak, alors que, de son côté, la SES a été compromise par les non français et néerlandais. Dans ces conditions, pourra-t-elle dépasser le simple dénominateur commun et se donner les moyens de l'action?

En conclusion, l'ouvrage nous rappelle que l'administration américaine a présenté un nouveau NSS en mars 2006, qui donne plus d'attention à l'égard des Etats forts tels que la Russie, la Chine, l'Iran ou l'Inde, de même qu'au lien transatlantique. La lecture des différentes contributions démontre cependant qu'Al Qaeda, la Chine, ou d'autres qui restent peut-être encore à déterminer, n'ont pas remplacé le rôle joué par la Russie pendant la Guerre froide dans le renforcement d'une politique transatlantique cohérente. Etats-Unis et Union européenne sont ainsi susceptibles d'avoir des vues et des priorités différentes, déterminées par leurs géographies, leurs cultures et leurs moyens, accentuant un fossé stratégique de plus en plus profond. Rien ne dit cependant que ce fossé peut faire des deux puissances des concurrents, comme le démontre le rapprochement qui a suivi la guerre en Irak. En ce sens, l'ouvrage se termine sur une note optimiste, considérant que les 
relations transatlantiques reposent toujours sur un «lien», que les deux bords auraient intérêt à institutionnaliser dès aujourd'hui.

Bertrand Vayssière

Maître de conférences à l'université de Toulouse II-Le Mirail

\begin{abstract}
Berthold RITTBERGER - Building Europe's Parliament. Democratic Representation Beyond the Nation-State, Oxford University Press, Oxford, 2005, 234 p. - ISBN 0-19-927342-1 (hb) - 54,00£.
\end{abstract}

In recent years some political scientists have rediscovered and explored the history of European integration. Andrew Moravcsik first utilized historical case studies for his rational choice examination of the intergovernmental negotiation of "grand bargains". In contrast, scholars adopting historical institutionalist and sociological institutionalist approaches to studying the European Union (EU) actually require historical depth. Historical institutionalists argue, in essence, that the creation and evolution of institutions over time creates "path-dependencies" for current and future constitutional and policy options. Sociological institutionalists contend that their historical experience influences the political behaviour of actors, as do their beliefs and values which evolve over time. Whereas these different competing institutionalist approaches are often based on rigid ontological and theoretical assumptions, Berthold Rittberger, in his study of delegation of powers to the European Parliament (EP), attempts to integrate them for explaining how this institution developed from a weak assembly to a co-legislator, with the Council, in many policy fields.

As a political scientist, Rittberger was puzzled to find that existing theories failed to explain the delegation of powers to the EP. Rational choice institutionalists like Mark Pollack at best admitted that their explanatory models did not cater for the increase in EP powers. After all, why should member-states give up powers to an institution that was not a "principal agent" and could not be easily controlled to guarantee policy outcomes in line with national preferences? To address this weakness in the existing political science literature, Rittberger has combined aspects of different "institutionalisms" with democratic theory. In a typical social science research design, his hypotheses outlined in two introductory chapters are tested in three case studies, the creation of the Common Assembly in the treaty establishing the European Coal and Steel Community (1951), the delegation of limited budgetary powers to the EP in the Treaty of Luxembourg (1970) and the extension of the EP's powers in the Single European Act (1986) and the Maastricht Treaty (1992). The analysis is largely based on published documents and primary sources, literature, a few interviews and some archival sources.

Rittberger argues that two main reasons account for the delegation of powers to the EP. The first is the concern of national political elites with preserving democratic procedures established at the national level within the newly created supranational polity. In essence, powers become transferred to the supranational 
level to enhance the member-states' problem-solving capacities (an argument in line with Alan Milward's historical approach). Decision-making in "Brussels" reduces the procedural legitimacy of decision-making, however, which requires compensatory constitutionalisation at the European level. Secondly, such legitimacy concerns become articulated in what Rittberger calls the "founding moments" of the creation of new supranational institutions or the deepening of integration in treaty revisions. He argues, in contrast to Moravcsik, for example, that procedural legitimacy concerns genuinely matter in processes of constitution-building, and that normative constraints influence the outcome of intergovernmental bargains. Diverging member-state preferences for tackling the procedural legitimacy deficit are primarily explained with different national traditions of democratic governance. Such preferences are not stable, however, and can be influenced by strategic rhetorical action to shape and define strategies for addressing the "democratic deficit". In this context, Rittberger draws on Frank Schimmelfennig's approach for explaining why France eventually agreed to the limited, but highly symbolic extension of EP powers in 1970, and countries like Britain and Sweden to the eventual extension of co-decision in the Treaty of Amsterdam (1997).

From the perspective of a historian, Rittberger's study (originally submitted as a $\mathrm{PhD}$ thesis at Oxford University) suffers from a limited source base and utilization of recent historical literature typical of a social science research design. Substantially, its main weakness is arguably the treatment of states as cohesive actors with cohesive preferences, however. National political actors operated under the same national constitutional set-up, but neither experienced nor judged its merits in the same way. At the time of the Schuman Plan, this became blatantly clear in the Belgian constitutional crisis over the "King Question". Similarly, the German Christian-democrats and Social-democrats had strongly diverging preferences for the constitutional set-up of the Federal Republic between a federal state with a relatively weak centre (in Bonn) and the centralisation of competences (in Frankfurt) to facilitate economic planning. Yet, Rittberger largely ignores the fragmentation of domestic preferences for European-level constitutionalization. Moreover, competing national actors also set up and utilized multiple transnational networks to support their particular constitutional preferences. Their democratic legitimacy strategies were not limited to the inter-state forum of treaty negotiations and bargains, but extended to parliamentary and public debates. These strategies were also influenced by the EP itself, once it had come into being, which acted for a long time primarily as a promoter of enhancing its powers, developing its own rhetorical strategies to foster this overarching objective. Finally, the constitutional preferences of multiple, not just national state actors, evolved over long time-spans, something that the case study approach cannot capture well.

Nonetheless, Rittbergers's book raises crucial questions about the historical evolution of the current EU. Historians can learn a lot from the stringent analysis of the process of delegation, and what might have motivated it, and address similar issues in their archive-based studies. In fact, the EP has been too marginal to 
contemporary historical research on the EU due to its limited formal powers until the Single European Act. The long debate about the Community's parliamentarization and the actual role of the EP in the political process, which appears to have been more important than the lack of formal powers would suggest, clearly deserve to be studied more in the future.

Wolfram Kaiser

University of Portsmouth

\section{Bertrand VAYSSIÈRE - Vers une Europe fédérale? Les espoirs et les actions fédéralistes au sortir de la Seconde Guerre mondiale, P.I.E.-Peter Lang, Bruxelles, 2006, 416 p. - ISBN 978-90-5201-353-4 - 34,90 / 38,40 €.}

Fruit du texte remanié d'une thèse soutenue en 2000 à l'Université de Toulouse le Mirail - Un groupe de pression européen: l'Union européenne des fédéralistes (1940-1956) -, l'étude de Bertrand Vayssière est consacrée à ce mouvement européiste qu'est l'UEF, tant d'un point de vue interne que dans l'horizon élargi de l'Europe et du monde. L'essence de cette organisation porte déjà en elle l'ouverture et enjambe les cadres nationaux. En effet, l'UEF, fondée en décembre 1946, se voit constituée $a b$ initio de divers mouvements fédéralistes conçus sur une base nationale variée. Elle représente, de manière inédite, une plate-forme commune aux fédéralistes. L'étude que lui consacre Vayssière, en plus d'être une mine de renseignements sur le groupement lui-même, éclaire d'un jour nouveau les grands débats et espérances qui traversèrent le cheminement du projet européen.

La genèse de cette nouvelle génération d'européistes - le deuxième conflit mondial et son milieu de la résistance - est d'abord envisagée. La guerre qui sévit est, pour les fédéralistes, la preuve de l'échec affligeant des États-nations. En plein conflit, des penseurs sont actifs, des mouvements sont formés, les fédéralistes multiplient activités et prises de position ('Manifeste de Ventotene', réunions européistes en Suisse, 'Déclaration des résistances européennes', etc.). Forts d'une croyance qu'il sera possible, à la Libération, de tout changer, ils veulent, dans un esprit révolutionnaire, enrayer le cycle guerrier en assurant à l'Europe un avenir fédéral.

$\mathrm{Au}$ lendemain de la guerre, tous les espoirs sont permis en ce qui concerne le futur supranational de l'Europe. Dans cette effervescence, les 15 et 16 décembre 1946 voient la création de l'UEF au siège du mouvement La Fédération à Paris. Douze formations issues de sept pays jouent ainsi, malgré leur diversité, la carte de l'union.

L'optique demeure, à cette époque, révolutionnaire et, afin de lutter pour le renouveau, l'UEF se doit d'être pétrie de militantisme. La volonté d'un engagement véritablement actif, couplé de recrutements et de campagnes d'opinion, est l'une des résolutions par lesquelles les fédéralistes tiennent à se démarquer de leurs prédécesseurs. Ils reprochent aux projets européistes de l'entre-deux-guerres leur inefficience; la conflagration de 1939-1945 en étant, à 
leurs yeux, le témoin patent. Un foisonnement de critiques fleurit à l'encontre de ce qu'ils considèrent comme l'élitisme, le verbiage ou les 'réalisations internationales stériles' de leurs devanciers. Les espérances ou sentiments européens antérieurs doivent désormais être transformés en militantisme efficace.

Avec la naissance de l'UEF, les fédéralistes tentent de faire connaitre leurs idées auprès des gouvernements et opinions. Durant cette nouvelle phase, les principaux congrès se tiennent - congrès qui, notons-le, occupent, d'une manière générale, une place fort importante dans l'ouvrage de Vayssière -, la période est à la découverte réciproque des divers mouvements, des personnalités s'affirment, avec pour corollaire certains différends idéologiques.

Cependant, malgré l'élan de la Libération et les aspirations qu'elle avait générées, les désillusions happeront rapidement les fédéralistes. Contrairement à leur croyance, le paysage politique n'est pas si ébranlé et les États-nations reprennent vie. De plus, la guerre froide va modifier complètement la donne mondiale et entraîner, dans son sillon, l'UEF vers d'inéluctables changements de cap. Le Fédéralisme se voit contraint de s'aligner sur l'un des deux 'grands'. Il abandonne, dès lors, son rêve d'État européen à l'échelle du vieux continent, comme celui d'Europe 'troisième force', pour se borner au cadre d'une Europe 'occidentale', alliée des États-Unis. Cependant, la menace que constitue le bloc de l'Est apporte de l'eau au moulin fédéraliste, ses représentants s'en servent, en effet, pour tenter d'unir les Européens, dans une réaction de défense. Ainsi, les visées révolutionnaires de l'époque de la résistance fondent pour se muer en un fédéralisme plus pragmatique.

L'ouvrage de Vayssière poursuit son parcours chronologique initié au cœur de la Seconde Guerre mondiale en traversant moult événements et réalisations d'importance, tel le Congrès de La Haye, en 1948, le changement de donne lié au plan Schuman, la mort de Staline, le combat de l'UEF pour la CED, ... pour l'achever à la fin de l'année 1956, année qui signe la scission de l'UEF.

Si la structure du livre suit un déroulement chronologique, les problématiques et questions transversales y sont privilégiées.

Une première ligne de force concerne le mouvement envisagé d'un point de vue interne. Son histoire propre, ses composantes, ses options doctrinales, les tensions qui le sous-tendent ..., sont des thématiques traitées au fil de l'ouvrage. Plate-forme commune, centre d'impulsion, l'UEF regroupe divers groupements fédéralistes sans les absorber. Ces derniers gardent une existence autonome, l'affiliation à l'UEF n'étant qu'une deuxième appartenance pour les adhérents des formations nationales. Le livre est, par conséquent, riche de renseignements quant à ces mouvements qui ont contribué à la fondation de l'UEF ou s'y sont joints par la suite: Movimento Federalista Europeo (le MFE créé pendant la guerre est, au niveau du fédéralisme européen, la branche la plus importante ne fût-ce qu'en termes d'activistes), Europa-Union, La Fédération, etc. La cohabitation entre ces formations éclatées ne sera pas toujours apaisée, il en va de même pour la relation entre les hommes qui les composent. Cette échelle individuelle est omniprésente dans l'ouvrage, en particulier, quant aux ténors de la cause fédéraliste: Altiero 
Spinelli, Henri Brugmans, Alexandre Marc, Henri Frenay, ... dont les rapports furent, eux aussi, pour le moins houleux. Loin de constituer un bloc homogène, l'UEF doit donc faire face à des tensions internes récurrentes de multiples natures, les divergences en termes d'option doctrinale, comme la dissension entre fédéralistes 'intégraux' et 'constitutionnels', n'y sont pas étrangères.

L'échelle européenne est également très riche dans l'étude. Si le milieu fédéraliste pâtit de son hétérogénéité, il est aussi confronté aux autres tendances de la nébuleuse européiste, plus ou moins divergentes de sa propre ligne de conduite (p.ex., celle constituée par les unionistes). Bertrand Vayssière étudie les contacts existants et les étincelles qu'ils peuvent produire, comme au sein du Mouvement européen, dont l'UEF est l'un des six fondateurs.

La dernière échelle est internationale et l'auteur met en exergue combien elle conditionne l'action des fédéralistes et l'évolution du mouvement. La Seconde Guerre mondiale tout d'abord qui en est la matrice, la Guerre froide ensuite qui balayera les rêves d'une Europe 'troisième force', le rôle des États-Unis, les relations franco-allemandes, etc. sont des événements cruciaux pour comprendre l'histoire de l'UEF.

Enfin, l'ouvrage se clôt par des annexes exposant clairement une série de données fort intéressantes. Des tableaux récapitulatifs des organisations membres de l'UEF, classées par pays, apportent, lorsque les informations furent disponibles à l'auteur, des renseignements sur le nom du président du mouvement, le nombre de sections, d'adhérents et de parlementaires. Trois clichés (1947 - à partir de 1950 - 1956) se suivent chronologiquement, permettant de saisir l'évolution relative à ces données. Deux autres tableaux chronologiques concernent la presse fédéraliste avec les indications suivantes: titre de l'organe périodique, mouvement dont il est issu, périodicité et directeur. Ensuite, une chronologie de l'histoire de l'UEF expose les éléments les plus importants et fixe les cadres chronologiques. Un index, in fine, a été constitué fort utilement, vu l'abondance de personnalités, d'organisations, etc. citées tout au long du volume.

Structuré, clair, rigoureux et fourmillant d'informations, le livre de Bertrand Vayssière est indispensable à tout qui veut pénétrer le monde complexe et bigarré des fédéralistes de l'après-guerre, leurs relations avec l'européisme et l'impact qu'a pu avoir l'échiquier européen et mondial sur le mouvement et sa doctrine.

Gaëlle Courtois Aspirante au Fonds de la Recherche Scientifique-FNRS Université Catholique de Louvain

Guy VERHOFSTADT - Les Etats-Unis d'Europe, coll. Voix politiques, éditions Luc Pire, Bruxelles, 2006, 67 p. - ISBN 2-87415-609-4 - 11,00 €.

Le livre du Premier ministre belge se veut être un «manifeste», à même de relancer une Europe en mal de projet depuis l'échec du TCE lors des référendums français et néerlandais. Conscient de l'affaiblissement de l'Europe face à des concurrents 
confirmés (Etats-Unis), et d'autres émergents (Chine, Inde), «l'ergotage» ne lui paraît plus un luxe que l'on peut s'offrir. C'est dire que ce court essai ne s'embarrasse pas de fioritures: la solution fédéraliste s'impose au fil des pages, autour d'un projet qui soit à nouveau commun (une «stratégie socio-économique communautaire»). Certains axes forts doivent être suivis dans le sens de «l'intégration», tels que la justice ou la politique étrangère, quitte à accepter pour de bon la thèse des «cercles concentriques», la partie de l'Europe prête à aller jusqu'au bout du processus existant déjà aux yeux de l'homme politique, correspondant à l'actuelle «zone euro».

Le thème de la crise européenne, si à la mode depuis un an, est repris ici par l'auteur, mais analysé assez froidement (c'est tout le mérite de Verhofstadt de laisser de côté les effets oratoires qu'il a pu un temps utiliser dans ses campagnes): que faire d'une Europe qui ne passionne plus les jeunes, qui n'apporte pas de réponse aux inquiétudes quotidiennes de la population? Il faut par conséquent se pencher sur les raisons de l'échec pour corriger une trajectoire de plus en plus faussée. En effet, il serait abusif, voire démagogique ou politiquement intéressé, de croire que les Européens ne veulent plus d'Europe ... C'est vrai dans un sens, mais l'interprétation de l'auteur est ici prise en défaut: d'après lui, le soutien indéfectible de l'opinion à l'idée européenne est prouvée par l'Eurobaromètre, et le principal défaut que les votants ont trouvé au projet de TCE est qu'il respire trop le compromis (p.14; autre simplification, qui a trait à l'histoire : la CED aurait été rejetée en France «sous l'influence [du seul] Charles de Gaulle», p.24)! Plus sensée est la démarche suivie par Verhofstadt pour comprendre les ressorts du non, et elle aboutit à une réponse moins simpliste que la précédente: l'angoisse et le doute ont une forte part dans ce rejet d'une Europe qui ne paraît plus être qu'un avatar, ou un fourrier de la mondialisation. Cet état atteint les couches les plus profondes de l'individu ainsi mis en danger, qui ont trait à l'identité. Dans ce constat, on peut dire que l'auteur fait preuve de courage, reconnaissant (et il s'agit d'un fédéraliste convaincu) que l'Europe ne peut pas exister, ou être comprise, en focalisant son action sur la seule réglementation. En ce sens, toute accélération ne peut provoquer qu'un décrochage, ce que l'ouverture des négociations avec la Turquie a confirmé.

Dès lors, l'Europe ne pouvant se surimposer, elle devra changer de projet et de philosophie, et accepter de faire certains choix. De toute manière, c'est vrai que l'évolution récente de l'Europe, multipliant les régimes d'exception, démontre que le projet est de plus en plus optionnel, ce qui dans l'immédiat traduit un affaiblissement de la notion de coopération européenne. Constat d'importance: l'Europe repose sur un déficit d'image, savamment entretenu par certains hommes politiques (l'auteur n'insiste hélas pas assez sur ce point, où aucun exemple, même tiré d'un lointain passé, ne vient étayer ses propos), qui encouragent les opinions à se retourner contre l'hydre bruxelloise. Tout cela entraine une inertie générale dont Verhofstadt, en tant qu'acteur, a été récemment témoin: l'échec retentissant du Conseil européen de juin 2005, le détricotage du Pacte de croissance et de stabilité, tout va dans le sens d'une indiscipline, sourdement encouragée par un devoir de résistance ou de désobéissance à une Europe présentée comme liberticide ou 
gaspilleuse. Or, le message du Premier ministre est positif: le premier devoir, qu'il signale dans son manifeste, est de dire la vérité, rappelant que l'Europe n'a jamais été, et n'est pas, un simple projet de libre-échange. Les exemples concrets de la PESC et de l'euro sont intellectuellement cohérents, mais rencontrent-ils la sympathie ou le soutien des Européens? Voilà une autre question à laquelle le manifeste ne s'embarrasse pas de répondre. De même, l'auteur ne pose-t-il pas les bases d'un débat biaisé en appuyant sur un parallèle «frappant» entre Etats-Unis et Union européenne (p.35; allusion maladroite prolongée par l'idée que le modèle américain «nous indique clairement la voie à suivre en Europe», p.40)? En tout cas, le basculement des premiers vers une formule entièrement fédérale, qui date de 1929 et des effets de la crise, a fort peu de chances de se reproduire pour l'UE, dans la mesure où la crise qu'elle expérimente depuis une dizaine d'années n'est pas strictement parallèle: elle est beaucoup plus politique, et pas aussi foudroyante que l'autre.

Cela dit, l'auteur continue sa diatribe contre la démagogie politique, n'hésitant pas à donner ici des exemples plus précis de ce qu'il ne faut pas faire (l'idée de directoire de Nicolas Sarkozy, de retour à la lire de la part de Roberto Maroni). Le but de la manœuvre est désormais d'allier le projet et l'efficacité, ce qui sous-entend un véritable partage des pouvoirs, idée qui rompt ici clairement avec l'euro-optimisme béat, et qui surprend même par sa précision: pour Verhofstadt, culture, sport, soins de santé, sécurité sociale, enseignement, gestion des services publics et appareil judiciaire doivent rester du ressort des Etats! Voilà qui est osé et rompt avec le politiquement correct européen, même si on peut révéler certaines contradictions dans le discours de l'homme politique belge (comment respecter une justice vraiment nationale quand on exprime le vœu qu'Eurojust devienne au plus vite une réalité, comme le dit Verhofstadt plus haut?). Toujours est-il que l'auteur propose cinq missions à la «nouvelle Europe»: mise en place d'une politique économique et sociale communautaire, avec un pilotage politique assuré par un «cabinet socio-économique européen», l'adoption d'un code de convergence tenant en compte les besoins et les réalités spécifiques à chaque Etat et une réforme des régimes fiscaux dans l'optique d'une meilleure compétitivité à l'échelle mondiale; politique technologique ambitieuse qui passe par un relèvement de la R\&D (Verhofstadt propose que le budget consacré soit le deuxième poste européen après l'agriculture) et l'adoption d'un véritable brevet européen (l'auteur confesse en passant le semi-échec de la stratégie de Lisbonne); création d'un véritable espace européen de justice et de sécurité, de manière à englober la lutte contre la criminalité (peut-être trop largement attribuée à l'Europe de l'Est) et le terrorisme en passant par une concentration des forces, qui ne nécessiterait pas la création d'un service européen de plus, mais permettrait la rencontre et le travail en commun d'acteurs compétents de chaque pays; création d'une diplomatie européenne, avec un ministre européen des Affaires étrangères ayant un réel pouvoir, un service diplomatique européen et un seul siège au Conseil de sécurité des Nations Unies; création d'une armée européenne, ce en quoi le ministre reprend un sujet qui lui est cher et où la Belgique, avec d'autres pays, a toujours été 
à la pointe face à une résistance par ailleurs signalée (voir le « pétard mouillée» que fut la proposition de créer un quartier général européen en 2003). Pour cela, l'Europe doit avoir les moyens de ses ambitions, et jouir d'une véritable autonomie budgétaire, qui ne passe pas par les cotisations nationales basées sur le PNB (en passant, l'auteur rappelle l'anomalie de certaines «ristournes historiques», qu'il semble vouloir remettre en cause).

Pour améliorer la visibilité de l'Europe, Verhofstadt propose également de repenser les institutions, en commençant par changer leur nom (exemples de la Commission ou du Haut représentant de la PESC) et à redéfinir leurs fonctions. Louable intention, mais qui fait oublier à Verhofstadt que les trois pouvoirs traditionnels de la théorie de la séparation propre à chaque Etat n'existent pas en tant que tels à l'échelle européenne (d'où la difficulté de «veiller à ce que chacun des trois pouvoirs démocratiques reçoive les compétences et les missions que l'on attend d'eux», p.64). Il y a donc un travail important à réaliser afin de parvenir à ce qu'il propose: un gouvernement européen d'un côté, un système bicaméral de l'autre!

Plus réaliste est la suite de son discours, où l'auteur semble tirer les conséquences des dernières campagnes, désastreuses pour l'UE. L'idée dominante est que l'Europe à 25 n'est pas possible à réaliser en l'état, d'où l'importance de reconnaître qu'il existe un «noyau dur européen» (p.66) correspondant précisément à la zone euro. Peut-on rappeler à Verhofstadt qu'il déplore lui-même le fait que certains des membres de cette zone, et pas des moins influents, se signalent par leur manque d'empressement à respecter les règles européennes édictées par eux et pour eux? Ne risque-t-on pas d'avoir du mal à obtenir ce «noyau politique» qu'il espère?

$\mathrm{Au}$ final, le manifeste de Verhofstadt nécessite une relecture, qui expurge totalement le reste d'idéalisme européiste du texte dont l'auteur s'est employé à décrire la nocivité. Son projet peut-il, comme il l'espère, séduire les jeunes générations? Il n'est pas certain que ces dernières soient à la recherche d'une rationalité politique qui concernerait plutôt le monde de la pratique politique communautaire stricto sensu (clarification des missions, lutte contre les doubles emplois, etc.). Il semble que Verhofstadt n'ait tout simplement pas répondu à ce qu'il a justement posé au départ de son exercice: l'angoisse et le doute sont deux maux qui sont corrosifs et plus à l'œuvre dans la mise en place de nombreux fantasmes anti-européens que l'absence de clarté politique que l'on peut reprocher à l'UE. On ne voit pas en quoi les mesures préconisées peuvent répondre à ce que les Européens expriment, en l'occurrence la recherche d'un refuge, qui effectivement pourrait être joué par l'UE, mais certainement pas dans les conditions actuelles. Il est évident qu'un référendum européen est une bonne idée pour répondre à une question aussi essentielle que l'adoption ou non d'une constitution européenne ..., mais il faudrait d'abord savoir, avant d'organiser un tel événement, si chacun des Européens est prêt, au préalable, à renoncer à un paysage politique qui lui est bien plus familier. En d'autres termes, le but ici préconisée ne consiste-t-il pas à substituer plus qu'à construire quelque chose de vraiment neuf? 
Pourquoi un gouvernement européen, créé du jour au lendemain, serait-il forcément légitime? Même question pour un parlement ayant de réels pouvoirs? Tant que l'on ne tiendra pas compte que l'idée de souveraineté ne se décrète pas, mais qu'elle repose sur une lente acceptation où sympathie et devoir d'obéissance sont étroitement mêlés, on continuera à contourner le problème européen face aux nations. La veine hugolienne signalée au début de l'ouvrage est peut-être désormais archaïque, si belle soit-elle, comme toute utopie qui a épuisé tous ses charmes face à une réalité tenace. Peut-être désormais devra-t-on abaisser les objectifs, et réclamer la formule modeste, mais plus accessible, des «Etats-nations unis de l'Europe»?

On doit tout de même retenir de cet ouvrage la courageuse entreprise du Premier ministre belge, seul homme politique de premier plan à avoir reconnu l'absence de sens de la démarche constitutionnelle proposée en 2005. Cette rupture avec l'«eurospeak» est déjà un premier pas, qui devra être suivi par d'autres, si l'on veut éviter que la vie politique européenne ne se situe que dans l'ordre virtuel.

Bertrand Vayssière maître de conférences à l'université de Toulouse II- Le Mirail

\section{Marie-Thérèse BITSCH, Wilfried LOTH, Charles BARTHEL (dir.), Cultures politiques, opinions publiques et intégration européenne, Bruylant, Bruxelles, 2007, 477 p. - ISBN 978-2-8027-2373-8 - 65,00 €.}

Fruit d'un colloque organisé à Luxembourg du 15 au 17 juin 2005, qui ne pouvait pas trouver mieux comme thème 15 jours après le double «non» franco-néerlandais au traité de constitution européenne (même si le colloque était programmé depuis de longs mois). A partir d'une multitude de cas, présentés par une variété de chercheurs très large, l'occasion était belle de se pencher sur cet éventuel «vouloir-vivre» européen, après lequel toutes les sciences humaines sont à la recherche. A l'heure où le «déficit démocratique» reproché à l'Europe débouche une nouvelle fois sur une sanction par l'opinion, que peut-on dire des sensibilités des différents peuples face à la construction européenne? Celles-ci semblent faites d'invariants, d'origine nationale, qui influent sur l'image de l'Europe, comme c'est le cas de la vision française, étudiée au niveau des dirigeants (Gérard Bossuat): des facteurs conjoncturels jouent, comme la guerre froide, mais également le désir de s'aider de l'Europe pour recouvrer une puissance peu ménagée par la Seconde Guerre mondiale. Cette vision, qui rapproche la IVe République de de Gaulle, continue sur un mode moins «lyrique» à partir de Pompidou, tout en évitant la question du cadre politique (intergouvernemental ou fédéral?), qui rebondit sans réponse jusqu'à nos jours.

Autre cas, celui de l'Autriche (Michael Gehler). Dans ce pays, le rêve impérial s'est dissous depuis longtemps, ce que montrent les sondages, où l'opinion s'avoue peu concernée par la politique extérieure. Après la demande d'adhésion (17 juillet 1989), certaines réticences se font jour, dans un pays qui, de par sa situation 
géographique et historique, a une sensibilité beaucoup plus forte aux événements se produisant à l'Est. Le référendum d'approbation, en 1994, est largement en faveur du «oui», mais un «oui» qui n'est pas fait que de bonnes raisons (l'auteur parle de «fuite en avant»).

Wolf D. Gruner s'interroge sur les résonances du débat fédéraliste chez les Allemands depuis la fin de la Seconde Guerre mondiale: ce dernier passe par le compromis de la Loi fondamentale de 1949, dont le préambule exprime le vœu d'une Europe unie. Ce débat a également des conséquences intérieures, qui met en œuvre, au quotidien, une collaboration étroite entre l'Etat fédéral et les Länder en matière de politique européenne. Ainsi, l'histoire récente démontre une certaine constance des Allemands, qui ont toujours été en faveur de solutions fédérales appliquées à l'Europe.

En ce qui concerne le Danemark et l'Europe (Sebastian Lang-Jensen), l'étude de la gauche de ce pays donne de curieux résultats. Au départ, la CEE est vue comme un instrument de l'Allemagne en particulier et du capitalisme en général, comprenant des pays qui ne partagent pas les «valeurs» des Danois. En conséquence, la gauche adopte une rhétorique indépendantiste, tout en appuyant les tentatives de créer une union entre pays nordiques, plus axée sur le consensus que sur l'intégration, mais qui toutes échouent. Sans surprise, la gauche se montre hostile lors du référendum de 1972. Depuis, nombreux de ses membres ont changé leurs points de vue, et c'est maintenant plutôt la droite qui est crispée sur la défense de l'identité danoise.

Alexander Reinfeldt étudie le cas de la Grande-Bretagne, où l'opinion n'a pas été aussi hostile que l'on peut penser. Celle-ci est plutôt versatile, partagée entre les sirènes du gouvernement britannique et celles de la Commission européenne (pour sa part, la CECA a installé un bureau à Londres dès 1956). Cela dit, la communication est plutôt à destination des élites de la part de cette dernière; quant au gouvernement britannique, il a tendance à ne pas adopter une politique constante d'information, utilisant l'opinion comme moyen de pression auprès de l'Europe.

Comment l'opinion publique se comporte-t-elle face aux élargissements? Pour l'opinion française (Anne Dulphy et Christine Manigand), ces derniers ont toujours posé problème. Molle et suiviste en 1972, l'opinion publique s'est montrée très rétive en ce qui concerne l'entrée des pays du Sud; l'hostilité est carrément déclarée à propos du dernier élargissement. Dans les mentalités, il existe une limite économique (et au fond culturelle) qu'il est difficile de franchir; de même, l'euroscepticisme est devenu plus fort à la fin des années 80 , avec pour sommet le 29 mai 2005. Les auteurs appuient d'ailleurs sur le manque de prise en compte par les élites de cette hostilité «annoncée».

Jürgen Elvert étudie plus généralement les différents referenda qui ont mis aux prises les opinions avec la question des élargissements. Différents sentiments s'expriment ainsi dans les pays qui accèdent: en Grande-Bretagne, l'argument est principalement économique, mais témoigne également de la peur de s'isoler, et de 
l'espoir que le pays prendra les commandes. Dans les autres pays étudiés, c'est le seul point de vue économique qui semble primordial.

Le «sentiment européen» n'apparaît pas vraiment dans tous les cas considérés, où la dimension politique est quasiment absente. Le cas polonais (Wojciech Prazuch) démontre un enthousiasme très fort de départ, même si le manque d'informations est patent. Au sein même de l'opinion, les différences sociales sont très fortes. Cela dit, le soutien à l'intégration n'empêche pas un certain pessimisme et la peur de se retrouver en «seconde classe». Pour la Hongrie (Balázs Hidvéghi), un retour à l'histoire rappelle que ce pays a depuis longtemps choisi la voie de l'occidentalisation (le fameux «dernier bastion de l'Ouest»). Cependant, même s'il est le premier a intégrer les structures internationales dès les années 80 , le rapprochement avec l'UE perd de son charme à mesure que l'entrée se fait attendre, la population passant par des phases d'enthousiasme et d'apathie.

Le débat à propos de la Turquie (Jürgen Nielsen-Sikora) souligne l'image très négative et stéréotypée que les Européens ont des ressortissants de ce pays. Quelles qu'en soient les conséquences, cette controverse représente pourtant un moyen pour l'Europe de se déterminer elle-même et de définir ses limites, réelles ou imaginaires.

Peut-on parler de corrélation entre les opinions publiques et la vie politique européenne? Jan-Henrik Meyer tente de définir ce qu'est une sphère publique européenne à partir de la réception du sommet de La Haye en 1969. Pour cela, il étudie six journaux allemands, français et anglais qui relatent les événements et décrivent les acteurs de ce sommet. Les visions de Georges Pompidou et de Willy Brandt sont contrastées, de même que les différentes politiques évoquées: la PAC est ressentie comme une exigence française, la Commission comme un organe technocratique mais indépendant, alors que la nécessité d'un progrès démocratique à l'échelle européenne est signalé par tous.

Comment les élites font-elles passer le message européen? L'Europe défendue par Valéry Giscard d'Estaing, étudiée par Michèle Weinachter, varie entre une vision «d'en-haut» (domaine réservé, politique «entre experts») qui peut rencontrer l'incompréhension du public, et une pédagogie plus fine (phase d'écoute de la Convention sur l'avenir de l'Europe), surtout à partir de 1989, où l'ancien président assume une vision très fédéraliste de l'Europe.

Birte Wassenberg étudie la campagne pour les élections européennes de 1979 en France et en Allemagne. Dans ce dernier pays, l'intégration européenne n'est pas l'objet de querelles, ce qui aboutit à un exercice politique consensuel, où prime l'intérêt national (Otto von Habsburg est le seul à surnager); du côté français, les querelles sont nombreuses, notamment à cause du problème de la supranationalité, et du retour du vieux démon allemand (Jacques Chirac, Michel Debré et leur liste «Défense des intérêts de la France en Europe»). Au final, l'auteur juge le «test assez décevant» dans les deux cas.

Vincent Dujardin prend le pouls de l'opinion belge, de la Libération jusqu'à 1979. Le pays, qui a abandonné depuis lors la neutralité, se montre dans l'ensemble favorable à l'intégration, mais sans bien connaître les différentes institutions, de 
même que tous les plans «européens» qui se succèdent. Le Marché commun paraît «très utile», mais on ne sait pas très bien avec qui on le fait. L'auteur fait le constat d'une dégradation, qui touche également la classe politique, que freine à peine la campagne de 1979.

Philippe Poirier, enfin, s'intéresse aux mouvements opposés à la «méthode communautaire». Les principales forces politiques présentes au Parlement européen n'ont pas de problèmes avec celle-ci. Parallèlement, les notions d'euroscepticisme et de souverainisme font une entrée remarquée dans le vocabulaire et les sensibilités politiques des différents pays membres de l'UE. La première peut être conservatrice ou extrémiste, la seconde est d'inspiration néo-gaulliste, mais a été récupérée par les libéraux (Margaret Thatcher), sans compter les mouvements régionalistes (FPÖ, Lega). Dans tout les cas de figure, ces ferments d'opposition font désormais partie intégrante du paysage politique européen, comme l'ont montré les différentes campagnes référendaires et leurs résultats négatifs.

Quelle est la part du facteur européen dans la culture des milieux sociaux? L'étude des syndicats français par Sylvain Schirmann montre des tendances lourdes et une évolution éclairée par l'histoire. Ces syndicats se sont montrés plutôt en faveur d'une législation sociale européenne, et d'un Parlement européen avancé. Cela dit, on ne peut pas parler de convergences entre les syndicats étudiés (CGT, FO, CFDT), qui ne se retrouvent que dans la critique de l'architecture institutionnelle.

Brigitte Leucht examine la politique de concurrence mise en place en Europe dans la foulée de la Seconde Guerre mondiale. Cette dernière a un sens depuis longtemps aux Etats-Unis, mais il a fallu l'imposer à des sociétés européennes réticentes, notamment à travers les autorités d'occupation et le Plan Marshall, que ce soit par la décartellisation en Allemagne ou l'accueil aux Etats-Unis d'experts et d'hommes politiques (parmi eux, Walter Hallstein). La CECA a été un moyen (articles 65 et 66) au service d'un véritable combat culturel.

Nigalé Bagayoko compare les opinions exprimées par les militaires français en poste au sein de l'UE et celles d'officiers n'ayant pas ces liens avec les institutions européennes. La différence est flagrante, se traduisant par une confiance plus large au sein du premier groupe, ainsi que plus de réalisme à l'égard de l'OTAN. A leur propos, l'auteur parle d'«immersion institutionnelle» et de «conversion».

Autre milieu, celui de l'administration européenne étudié par Katja Seidel. Le problème du recrutement est jugé si important qu'Hallstein lui-même le prend en charge. Les difficultés de départ (1958-1962) sont évoquées: il s'agit de constituer une administration indépendante, tout en subissant les pressions des Etats. L'auteur parle d'un véritable «esprit de corps» à propos des fonctionnaires A des Directions Générales (DG) concurrence et agriculture, eux-même aiguillonnés par le souvenir de la guerre, la sensation d'être des «pionniers» et l'effet d'entraînement des commissaires charismatiques à la tête de ces deux DG.

L'ouvrage se termine sur le thème de la transmission de l'idée européenne et de l'émergence d'une identité européenne. Luc Blanchart étudie l'enseignement de l'Europe 
en Belgique, et parallèlement le sentiment identitaire des Belges. Les résultats sont différents d'une communauté à l'autre, mais le constat est sans appel pour l'ensemble du système et de ses acteurs: l'enseignement de l'Europe est trop tardif, trop peu axé sur le politique, et démontre un manque d'intérêt et d'audace de la part des enseignants.

L'étude de courts-métrages et de films documentaires pendant les années 50 par Gabriele Clemens démonte les rouages d'un travail de relations publiques à la recherche d'un public transnational. Les donneurs d'ordre de ces films sont essentiellement américains, par le biais du Plan Marshall (plus de 200 entre 1948 et 1953), mais ils peuvent également être issus de mouvements militants (exemple du Banquet des Fraudeurs de l'Europa-Union) et de la CECA. Ces films insistent tous sur le thème de la guerre comme expérience commune, attribuant, parfois lourdement, une «faute originelle» aux différentes nations. L'Europe culturelle ne joue dans l'ensemble qu'un rôle secondaire: il s'agit d'une Europe de la prospérité, floue politiquement.

Enfin, l'étude du patriotisme constitutionnel par Muriel Rambour rappelle l'origine proprement allemande du terme (Dolf Sternberger). Ce dernier insiste sur la dimension démocratique qui entoure une définition très politique de l'Europe, un peu à l'image des déclarations de Copenhague de 1973 ou de 1993. Qu'en est-il de la part affective propre à tout attachement «patriotique»? Peut-on être d'accord avec la position de Habermas et de Derrida qui estiment que la position des Européens sur l'Irak correspond à un nouveau départ pour l'idée d'appartenance politique qui devrait les rassembler? Le débat reste ouvert, mais, depuis peu, on peut dire qu'il s'est plutôt focalisé sur la défense des identités nationales face à la dilution «orchestrée» par l'UE.

Ainsi donc, la politisation de l'Europe et l'européanisation du politique ne sont pas des faits démontrables, relevant plus, dans l'immédiat, d'intentions ou d'expériences à caractère limité, que d'un processus général en cours. C'est le mérite de cet ouvrage de vérifier que la discussion est ouverte à tous, partout en Europe, et qu'elle permet de revisiter certaines certitudes. Nous ne sommes plus à l'heure des tabous et des «théologies européennes», car, pour reprendre Denis de Rougemont (Robert Frank), «l'Europe n'est plus une question de vie ou de mort». Même si ce constat peut soulever une part d'inquiétude, il doit nous rappeler que jamais les Européens ne se sont interdits les voies du recommencement qu'offre tout débat ouvert, surtout en ce qui concerne un projet qui doit rester collectif.

Bertrand Vayssière

Maître de conférences à l'Université Toulouse II-Le Mirail

Michel DUMOULIN (dir.) - La Commission européenne, 1958-1972. Histoire et mémoires d'une institution, Office des publications officielles des Communautés européennes, Luxembourg, 2007, 642 p. - ISBN 978-92-79-05495-2 - 30,00 €.

Une institution peut-elle avoir une mémoire? Qui plus est une institution elle-même issue du néant, et «qui [doit] inventer [sa] route au fur et à mesure qu' [elle avance]»? La Commission européenne est une création des Traités de Rome, où la négociation l'a emporté sur l'enthousiasme, et où la prudence a primé sur l'audace. 
Le résultat est curieux: de nombreux objectifs, mais aussi une multitude de garde-fous. Face aux Etats qui ont démontré à cette occasion leur «obstination» à vivre, un organe est créé, qui n'aura pas les coudées franches de la Haute Autorité de la CECA, mais qui a la possibilité de représenter un «intérêt commun», qu'il reste à définir et à défendre.

Comment cet organe nouveau va-t-il se comporter? Quelles vont être les réussites et les handicaps, politique par politique? Ce sont ces questions que compte aborder ce passionnant ouvrage, qui applique pour la première fois à une institution européenne ce qui a déjà été fait par ailleurs auprès d'autres collectivités de nature politique: la collecte de témoignages, afin de faire connaître le système de l'intérieur. Qui sont les témoins? De vrais «Européens»? Ce terme-là ne signifie pas grand-chose, du moins au départ. Des pionniers? Le vocable paraît plus juste, et légitime le travail entrepris par la multitude d'historiens de toutes nationalités qui ont œuvré sous la direction du professeur Michel Dumoulin, avec le soutien de la Commission Prodi. Cette entreprise a un intérêt historique immédiat: simple question de générations, les premiers acteurs de la CEE sont aujourd'hui décédés ou bien à la retraite. Ceux qui ont été retenus pour cette étude (120 anciens fonctionnaires au total), dont les témoignages ont été collectés pendant trois années, ont occupé différents postes, plus ou moins exposés, pendant une période cruciale, celle des Six (1958-1972). Pourquoi ce temps-là? Il ne faut pas chercher au-delà des explications fournies en début d'ouvrage et que nous avons déjà explicitées: fugit tempus! La recherche n'en est pas moins favorisée par ce travail original: le témoignage oral est une source de choix, surtout pour une histoire qui cherche à dépasser le cadre parfois trop asséché de l'institutionnel (signalons que ces sources vont elles-mêmes être déposées aux archives de Florence). Simple question de sauver un «patrimoine» (p.36) d'un nouveau genre? Cette histoire des «premiers temps», des audaces bientôt balayées par la geste gaullienne, puis par le premier élargissement, ne relève-t-elle pas également de la nostalgie?

Plusieurs articles insistent, à l'appui des témoignages, sur le joyeux désordre de départ, à une époque où la Commission n'a même pas de siège fixe. Joyeux en apparence: dès l'origine, la lutte est à couteaux tirés entre cet «exécutif» de l'Europe venu de nulle part, et les différentes chancelleries des Six, qui verraient d'un bon œil la mise au pas de la Commission par le Coreper, né simultanément. Pourtant, les objectifs affichés sont ambitieux, élastiques grâce à ce «traité-cadre» qu'est celui de Rome: suppression de toutes les entraves au marché intérieur, nouvelle politique de développement agricole, politique commerciale commune, politique de la concurrence, politique d'aide aux pays en voie de développement. Autant dire que la Commission est dès le départ un creuset où s'entrecroisent les idéaux et les expériences des différents acteurs, sur des dossiers avec lesquels tout le monde, suivant sa propre histoire, n'est pas forcément familier. S'il est difficile de parler d'une «culture politique» spécifique, les différents articles permettent d'évoquer page après page un laboratoire en perpétuelle ébullition. On doit également écarter l'accusation de technocratie qui est si vite lancée contre ces pionniers de l'aventure communautaire (et leurs successeurs), les ravalant au 
simple rôle d'exécutants sans âme d'une politique commerciale et libérale, ou (c'est plus révélateur) d' «empêcheurs de tourner en rond». Dès le départ, le ton est donné, en particulier par le premier président, Walter Hallstein: derrière la technicité, derrière l'économie, il y a un projet d'ensemble, encore ténu et qu'il n'est d'ailleurs pas de bon ton d'évoquer encore à haute voix, celui d'inventer une nouvelle société, plus grande et plus juste, une société qui ne retient pas de l'Europe que l'idée d'harmonisation et de productivisme, mais également ses valeurs et son humanisme. Hallstein croit en la force motrice du développement, et la Commission doit être le dépositaire de cette «logique matérielle».

L'ouvrage est lui-même partagé en deux parties. La première s'intéresse plus particulièrement aux acteurs et à la difficile naissance de la Commission, dans un contexte où toutes les menaces n'ont pas été levées: propositions du comité Maudling, démissions successives des président et vice président de la Haute Autorité, bientôt l'arrivée de Charles de Gaulle au pouvoir. Après les travaux du Comité intérimaire (qui devient le Coreper), la Commission peut voir le jour, et son président désigné le 7 janvier 1958: ce sera l'Allemand Walter Hallstein. Cet homme a une haute idée de ce que doit être l'intérêt général de la CEE, et s'emploiera à le défendre avec une équipe restreinte de neuf commissaires. Il est vrai que le traité donne à la Commission un rôle capital, qui est celui d'être le «moteur de l'intégration» (capacités d'initiative, d'exécution et «gardienne des traités»). Cela ne suffit pas, étant donné la mauvaise volonté d'Etats qui se sentent dessaisis de politiques essentielles: une guérilla oppose le plus souvent les membres de la Commission aux administrations nationales. Très conscient de son rôle et souhaitant le faire savoir, Hallstein met en œuvre un protocole communautaire qui fait bouillir de rage le général de Gaulle (qui saura s'en rappeler). La crise de la chaise vide elle-même est l'occasion de voir s'affronter les deux hommes. Sa résolution, qui passe par la rencontre des 28 et 29 janvier, à Luxembourg, débouche sur le trop fameux «compromis», texte extra-judiciaire qui est d'abord une délibération formelle non faite pour entrer dans les traités, un «accord sur le désaccord» pour reprendre les termes de Walter Hallstein. Elle annonce une coupure dans la jeune histoire européenne à tous les points de vue, le triomphe de l'intergouvernementalité et, comme conséquence immédiate, la défaite de la pensée européenne de Hallstein, remplacé par Jean Rey en 1967, qui n'aura jamais l'étoffe et la marge de manœuvre de son prédécesseur. C'est le début d'une semi-paralysie de l'intégration européenne, mais également la vérification que, malgré sa rhétorique sur la souveraineté, la France ne pouvait se passer de l'Europe.

Certes, on a quitté l'époque des approches «théologiques» (Raymond Barre) de la question européenne, pour s'atteler à des tâches plus terre à terre, avec une institution qui s'est agrandie suite à la fusion des exécutifs (en passant, on se rend compte sans surprise que la Commission de la CEE est la grande gagnante de cette opération, surtout par rapport à l'Euratom). Ces tâches ont été accomplies par des hommes, que certains chapitres nous font mieux connaître, notamment à l'aide des témoignages toujours judicieusement utilisés: Jean Rey, Franco Maria Malfatti, le 
président-«météore», le commissaire Sicco Mansholt, très présent dans le paysage bruxellois, par son activisme, ses coups d'humeur et sa longévité, le secrétaire exécutif puis général de la CEE Emile Noël, le «dixième membre de la Commission», sans parler du «onzième», le directeur du service juridique commun, Michel Gaudet. A travers ces deux derniers, ce sont les membres anonymes mais récurrents des appareils bruxellois qui nous sont mieux présentés, leurs évolutions, leurs convictions (car l'étude, chapitre après chapitre, démontre que l'engagement n'est pas vain chez ces premiers fonctionnaires): directeurs généraux ou assistants, l'organigramme (si surveillé par Hallstein) répartit les tâches, ayant toute son importance dans la conduite que s'est imposée la Commission pendant toute cette décennie. Ce corps des fonctionnaires européens, on le découvre encore un peu mieux dans des chapitres consacrés à leur installation à Bruxelles: prises de fonction, rencontres et socialisation, motivations, scolarisation des enfants, bref, l'aspect humain de ces acteurs d'un nouveau genre et de tous horizons, dans un lieu qui très rapidement se prête au jeu et se coule parfaitement dans le rôle de capitale «européenne» (même si la question officielle du siège mettra du temps à être réglée).

Les politiques sont traitées les unes après les autres dans une seconde partie, avec leurs succès et leurs échecs. L'importance de la notion de marché commun est rappelée d'emblée, comme étant le socle du projet communautaire, et se présentant comme la raison de vivre et d'agir de la Commission (élimination des contingents et des droits de douane, élaboration d'un tarif extérieur commun). Le succès est tel que le calendrier arrêté au départ est finalement anticipé. En ce qui concerne la politique de la concurrence (inédite pour la plupart des pays européens), il s'agit de surveiller des habitudes qui ont été trop fréquentes dans le passé, notamment les ententes ou les aides d'Etat. Parallèlement, le phénomène de concentration est encouragé du moment qu'il est européen: pas facile à l'époque où chaque Etat veut mettre sur pied ses propres «champions nationaux». Les péripéties de la PAC sont mises en avant, rappelant l'importance de la conférence de Stresa, où Mansholt a su jouer un rôle positif dans un véritable bouillon de culture d'où naîtront peu à peu les règles de la première politique communautaire. En ce qui concerne les relations extérieures, la Commission commence à se faire connaître, notamment par le biais des négociations commerciales, et des représentations, pas toujours bien vues par les Etats, qu'elle ouvre un peu partout dans le monde; le même mode d'affirmation peut être signalée, en fin de période, la question de l'élargissement.

Intéressant également de noter les différences qui peuvent exister de pays à pays en ce qui concerne l'aide aux pays en développement, le passé colonial de certains des Etats membres jouant ici un rôle considérable. On peut aussi observer les tentatives d'une politique économique et monétaire ambitieuse, avec la création d'un Comité de politique conjoncturelle et l'effort d'assurer une meilleure coordination (plan Barre du 12 février 1969). Certaines politiques paraissent d'emblée difficiles: la politique régionale qui n'est qu'un simple encadrement, celle des transports, dans un domaine qui reste un «bastion des nationalismes», de même que l'Europe sociale. Concernant ce dernier cas, il ne faut pas oublier les 
objectifs strictement économiques des Traités de Rome, dans une période de croissance qui semble être sans fin. Quant à la politique industrielle, elle n'a même pas été mentionnée: on peut évoquer certaines actions sectorielles, comme dans les chantiers navals ou l'industrie textile, mais encore très modestes, malgré les débats intenses provoqués par un best-seller comme Le Défi américain de Jean-Jacques Servan-Schreiber. Le problème est le même concernant l'énergie, qui plus est dispersée entre les trois communautés européennes, ou pour l'action en matière de recherche et technologie. En revanche, la politique de l'information démontre qu'il y a une quête de l'opinion dès le départ, avec certains noms récurrents (Jacques-René Rabier, François Fontaine, François Duchêne, Emanuele Gazzo), ainsi que la recherche d'une meilleure visibilité (institution d'un porte-parole de la Commission). Cependant, les contacts privilégiés restent ceux qui sont noués avec les journalistes et les lobbyistes.

Un livre précieux, lui-même symbole d'une coopération réussie. Outre les articles qui offrent une présentation exhaustive des hommes et des politiques, on trouvera dans cet ouvrage une chronologie complète, les organigrammes successifs, le détail des sources (en particulier les archives orales, qui sont autant d'instruments de travail à exploiter), ainsi qu'une bibliographie très complète. Les documents, souvent inédits, qui parsèment les différents articles en rendent la lecture plus agréable. La preuve est faite que la Commission n'est pas qu'une institution que certains s'acharnent à trouver «technocratique», mais une aventure humaine engageant des personnes de chair et de sang, et qui toutes se retrouvent à Bruxelles pour inventer les bases d'une communauté inédite. A quand le même travail pour une période plus proche de nous?

Bertrand Vayssière

Maître de conférences à l'Université Toulouse II-Le Mirail.

Antonio VARSORI (ed.) - Sfide del mercato e identità europea. Le politiche di educazione e formazione professionale nell'Europa comunitaria, Franco Angeli, Milan, 2006, 219 p. - ISBN 88-464-7981-5 - $18.00 €$.

In recent years, the history of European integration has witnessed a surge of attention on European Economic Community/European Union (EEC/EU) policies, and in particular on the policies that have had a growing impact on the evolution of European society. This new interest of historians is a consequence of the increasing political competences that the EEC/EU has gained since the Seventies. From that time, the EEC/EU could not only be conceived as a mere economic entity because it has widened its powers to other relevant policy fields (i.e. research and development, social cohesion, environmental policies, etc).

The book under review, edited by Antonio Varsori, pays special attention to vocational education and training (VET) policies and to their emergence in the European Community and in some international organizations, such as the United Nations Educational, Scientific and Cultural Organization (UNESCO). This book 
is one of the results of a wider research project coordinated by the editor, which aims to illustrate the development and impact of VET policies in the different stages of European integration, as well as the role played by the EEC/EU institutions in this field and the activities run by the European Centre for the Development of Vocational Training (Centre européen pour le développement de la formation professionelle - Cedefop) between its birth in 1975 and the Nineties.

Varsori's chapter presents the general framework of VET policy evolution. He shows that in the "Europe of the Six" vocational and education training was first considered as an aspect of social measures to be implemented in order to meet production and labour market requirements. This approach was renewed under the particular climate of social unrest of the early Seventies, which was exacerbated by the demands of the student and trade union movements. However, while the EEC institutions' interest in VET was rapidly increasing, the member states' governments expressed mounting opposition because - as Varsori argues - they were eager to preserve their exclusive competence in the domain of education. The difficulties encountered in the establishment of Cedefop in 1975 were a clear example of the ongoing dispute between the Commission and the European Parliament on one side and the Council of ministers on the other side. While the Commission conceived Cedefop as a useful support to improving Community action in education and social fields, the Council tried to keep the new agency under its control in order to restrict Cedefop's functions to studies and research activities, without the possibility of influencing national education policies.

Despite these limits, Cedefop has played its own role in VET. This book helps to reconstruct Cedefop's activity in facilitating transnational cooperation in the field of VET policies, as it encouraged EEC/EU institutions and the member states to face central issues in the promotion of socio-economic development, such as the recognition of higher education and professional qualifications throughout Europe, the management of new technologies, and opportunities for young people and women to participate in the labour market. To this end, Marina Cino Pagliarello focuses on the Study Visit Programme for experts and people in charge of vocational training that was first promoted by Cedefop in 1985. Another central contribution to the Europeanization of educational policies is presented by Emanuele Torquati, whose chapter examines the birth and the evolution of the "Jean Monnet Action". As Torquati says, the "Jean Monnet Action", which started in 1989 with the support of the president of the European Commission Jacques Delors, became the "fulcro" - the hub - of a more complex Community strategy aiming at the expansion of European studies in the academic world. This project proved to be successful and contributed not only to creating a direct link between the Commission and the Universities but also to establishing Chairs and Modules especially dedicated to the teaching of European studies in different disciplines. The Study Visit Programme and the "Jean Monnet Action" - to which we must add Erasmus and Socrates Exchange Programmes - illustrate the widening of EEC/EU horizons from a solely economic dimension to become a more socially oriented entity. In this process, a key role was played by the European Parliament, which has 
always tried to push other institutions and national governments to face education issues within the Community framework. The chapter by Simone Paoli is of particular interest to historians because it clearly illustrates from a diachronic perspective the Parliament's long-lasting struggle to stress the importance of facing the common problems related to vocational education and training that arose with the creation and the concrete functioning of the common market.

The volume also sheds some light on the activities of international organisations, paying special attention to UNESCO's actions in the Sixties. In particular, Sara Banchi emphasizes the project realized in the Ivory Coast between 1961 and 1967 to create a centre to provide vocational training in the rural domain and to train permanent teaching staff in the region. This case study gives an important insight into the progressive qualification of VET as an investment in human capital. This is a sign of the growing awareness of the importance of VET as an essential tool to increase competitiveness and material growth, as well as to equip people with the widest possible range of skills and competences. In this sense, nowadays VET policies are considered to be a fundamental pillar of the Lisbon Strategy because - as Elena Mainardi's chapter reports - they play a central role in enhancing the "knowledge society" model the EU has decided to implement in order to successfully face global market challenges. In this sense - the book seems to suggest - VET may also contribute to shaping the elusive concept of European identity, by focusing on the concrete achievements of the EU that have improved opportunities for European citizens in their lives and work.

This volume deserves recognition for its achievements. It constitutes an original contribution to current historiography in both its subject matter and methodology. It manages to clearly illustrate the inclusion of VET policies in the Community framework and in doing so it deals with a subject that had not yet been fully investigated from a historical perspective. The methodology is also interesting because, by taking into consideration several actors involved in vocational and education training (international organizations, European institutions, national governments, etc.), the book gives an idea of the multilevel interaction that takes place in the implementation of many other important EU policies.

Laura Grazi

CRIE - University of Siena

Marc JOLY - Le mythe Jean Monnet. Contribution à une sociologie historique de la construction européenne, CNRS éditions, Paris, 2007, 238 p. - ISBN 978-2-271-06575-9-15,00€.

Cet ouvrage balance entre, d'une part, une réflexion sur le mythe et ses origines, appliquée à la construction européenne au travers de son plus célèbre représentant, et, d'autre part, une prise à partie politique contre cette même construction au nom d'un engagement militant transparent. Il n'est pas mauvais de rappeler que l'auteur est militant du MDC de Jean-Pierre Chevènement, et qu'il a déjà fait paraître un livre sur «le souverainisme» (Guibert, 2001), dont certains ont souligné l'apport, et 
d'autres le parti pris. Il faut de plus insister, ce que fait d'ailleurs Marc Joly (bien qu'à la fin, p.202), sur le fait que ce livre a été écrit dans une ambiance (et dans un but?) polémique, au moment de la campagne autour du Traité constitutionnel européen.

L'ouvrage commence par une allusion très marquée au traité modifié, présenté comme un complot fomenté «d'en haut»: le ton est donné, en particulier par la page 11, où le mot «élite» (ou sa déclinaison «élitaire») apparaît cinq fois, appliqué à ce que l'auteur, dans une approche très systémique, nomme le «pouvoir-Europe», nouvel avatar de ce que certains ont pu appeler «l'eurocratie». Il faut d'emblée souligner une contradiction dans ces propos: peut-on, comme le fait Marc Joly, souligner l'idée d'une «crise de type idéologique» à propos de l'Europe (p.12), et reconnaître, à plusieurs reprises, qu'il y a autant de visions de l'Europe que de pays membres? De même, le consensus, présenté comme la marque de ce pouvoir-Europe, est-il toujours à l'avantage de celui-ci? Le compromis de Luxembourg n'est-il pas une forme de consensus qui a d'abord eu pour but d'éviter toute avancée de l'intégration européenne? Mais non, la thèse est posée: l'Europe, manquant de légitimité, comploterait contre la démocratie. La démonstration se fait cependant au mépris de toute leçon historique, faisant par exemple abstraction des débats sur la CED et de leurs effets sur la construction européenne. Le travail de Joly consiste d'abord à stigmatiser le pouvoir autonome engendré par la CEE/UE, en s'appuyant sur une approche très politique (p.24), où les «citoyens» auraient des intérêts opposés à ceux de «l'Europe» (qui, tout au long de l'ouvrage, ne sera jamais vraiment définie). Il s'agirait alors pour cette dernière, forcément anti-démocratique, de «vendre» son projet.

D'où Norbert Elias, sur lequel notre auteur s'appuie pour faire la «chasse aux mythes». Dans cette optique, Monnet paraît l'homme idéal en tant que «M. Tout-le-Monde» (p.26: entre parenthèses, n'est-ce pas une garantie de démocratie que M. Tout-le-Monde soit un modèle à suivre, plutôt qu'un improbable Sauveur?), démiurge et créature de l'Europe (et pourtant, ce que l'auteur oublie, panthéonisé en France). Le pauvre homme est bombardé «mythe élitaire» (p.34), «Américain» (dans la note 18 p.45), et, plus ambigu encore, «Français dénationalisé» (p.75). Il n'a même plus de secrets de conscience, puisque Joly arrive à savoir quand Monnet «n'est pas forcément sincère» (p.194)!

Ces formules creuses, à l'emporte-pièce, continuent dans la partie intitulée «le civilisateur», où l'auteur démonte le «complot mythologique» qu'il a éventé au départ de son ouvrage. Cela dit, son propos commence avec une nouvelle légèreté historique: Monnet n'a pas été démis de son poste de président de la Haute Autorité (p.35), mais l'a quitté volontairement, pour se consacrer au Comité d'action qu'il a créé dans la foulée. Certes, il est juste d'évoquer le fait qu'il se range derrière un projet de CEE qu'il n'a pas voulu, mais qu'il légitime par son «magistère sacré». L'auteur oublie seulement de préciser que ce n'était pas vraiment le fruit de sa propre volonté ... Parallèlement, le mythe de la CECA, qui aurait été savamment entretenu par Monnet à travers ses Mémoires, ne servirait qu'à couvrir un «marchandage diplomatique classique entre l'Allemagne et les Etats-Unis» (p.50). 
La CECA ne serait en effet ainsi qu'un écran de fumée masquant «la domination écrasante du capitalisme américain» (p.67), et celle de technocrates que Joly illustre à l'aide d'une seule citation volontairement provocatrice de Pierre Uri (p.69). Dans cette optique, même le Plan institué par le gouvernement français ne relève que d'une volonté de domination technocratique (n'est-ce pas de Gaulle qui l'a institué?). L'auteur reconnaît pourtant lui-même que si cette volonté a existé, elle a été étouffée par la volonté des Etats (p.81, p.87, encore p.158), et que Monnet se donne avant tout «l'illusion» de contrôler le processus, avec son Comité d'action. L'essentiel est qu'il légitimerait le processus européen alors en cours aux yeux des Européens: mais comment, puisque l'on sait que ce Comité était à vocation justement très élitiste?

Le mythe Monnet fut celui d'un «despotisme éclairé» (p.105), définition plus juste, pour une fois bien illustrée (p.196); mais n'est-ce pas ce que disait déjà Hubert Védrine de la politique européenne de François Mitterrand? Condamner rétrospectivement cette tendance relève d'une part de l'anachronisme, et d'autre part sert avant tout une volonté de polémique qui se drape d'atours scientifiques vite envolés: pourquoi Joly veut-il absolument lier l'œuvre de Monnet à la pensée de Norbert Elias (il parle même d'un dialogue qui s'installerait entre eux p.181, p.186)? Ne veut-il pas remplacer un mythe par un autre? Il semble qu'il ait besoin d'un cadre rassurant, que chaque chose soit à sa place (voir sa propre définition de l'européisme, p.147), quitte à forcer le cadre. Elias a étudié les Capétiens et leur domination progressive en France: le parallèle avec Monnet peut-il tenir? On s'inquiète quand l'auteur avance une explication «capitaliste» à la première entreprise (p.130), qui de plus a tous les aspects du darwinisme social. De toute façon, n'ayant pas peur de la contradiction, l'auteur trouve Monnet trop rationnel pour soutenir la comparaison (p.138)!

Dans l'ensemble, cet ouvrage est moralisant, et trop engagé politiquement. Il est plein de considérations très générales sur le fonctionnalisme, qui masquerait une confiscation voulue du pouvoir, et n'arrive pas à entrer dans la psychologie, ni du personnage principal, ni de l'opinion, ici habilement orchestrée pour la faire entrer dans le cadre rassurant d'une thèse, qui, après examen, ne vaut pas plus qu'une charge politique sans grande envergure. L'auteur voit le complot européen partout, ce qui lui permet de lier les «forfanteries» d'hier avec celles d'aujourd'hui, et de régler ainsi ses comptes avec le TCE, et autres prétendues tentatives d'étouffer le principe de citoyenneté. Décidément, Jean Monnet a bon dos ...

Bertrand VAYSSIÈRE

Maître de conférences à l'Université Toulouse II-Le Mirail

N. Piers LUDLOW - The European Community and the Crises of the 1960s. Negotiating the Gaullist challenge, Routledge, London and New York, 2006, 269 p. - ISBN 0-415-37594-0 - 103,99 €.

In der Erforschung der Geschichte des europäischen Integrationsprozesses zeichnet sich in den letzten Jahren der Trend ab, die Integration bestimmter Politikbereiche 
mit einem multinationalen Ansatz zu untersuchen. Im Blickpunkt steht dabei nicht mehr ein einzelner nationaler Akteur, sondern die Autoren untersuchen das Zusammenspiel der verschiedenen an der Integration beteiligten Länder und der europäischen Institutionen. Diesem Ansatz ist auch die Studie des renommierten Londoner Historikers N. Piers Ludlow verpflichtet. Ludlow geht in seiner Studie jedoch über den sektoralen Ansatz hinaus und nimmt den Integrationsprozess der 1960er Jahre in seiner ganzen Breite in den Blick. Er rekonstruiert dabei die EWG-Verhandlungen überwiegend mit Quellen aus den Archiven des Ministerrats und der Kommission der EWG. Mit Hilfe des Aktenmaterials aus den nationalen Archiven der Mitgliedstaaten versucht Ludlow zu analysieren, warum die einzelnen Regierungen bestimmte Positionen in den EWG-Verhandlungen vertraten.

Im Mittelpunkt des in 7 Kapitel aufgeteilten Werks steht der Umgang der EWG mit der Europapolitik des französischen Staatspräsidenten Charles de Gaulle zwischen 1963 und 1969. Laut Ludlow lassen sich die Auseinandersetzungen in der EWG der 1960er Jahre auf drei Grundkonflikte zurückführen, die ihre Wurzeln in den vagen Bestimmungen des EWG-Vertrags hatten. Zum einen musste präzisiert werden, wie und mit welchen Prioritäten das politische Programm des EWG-Vertrags umgesetzt werden sollte. Zum anderen musste sich das institutionelle Gleichgewicht noch finden. Außerdem musste die Frage ausdiskutiert werden, wer Mitglied in der EWG werden konnte und wie sich der Erweiterungsprozess vollziehen sollte. Diesen Grundkonflikten spürt Ludlow in seiner chronologisch angelegten Studie nach.

Ausgangspunkt ist dabei das Veto de Gaulles gegen einen britischen EWG-Beitritt vom Januar 1963. Die daraus resultierende Krise konnte relativ rasch überwunden werden, da sich alle Beteiligten von Fortschritten in der Integration Vorteile erhofften. Insbesondere konnte man so dem französischen Partner vor Augen führen, wie stark auch Frankreich auf die EWG angewiesen war. Dabei half der ambitionierte Aktionsplan, den der deutsche Außenminister Gerhard Schröder im April 1963 im Ministerrat vorstellte. Wie Ludlow betont, habe Schröders umfangreiches Programm jedoch nur Akzeptanz gefunden, weil es der Luxemburger Ratspräsidentschaft gelungen sei, das Papier so zurechtzustutzen, dass es für alle annehmbar gewesen sei.

Anschließend schildert Ludlow vor allem die Auseinandersetzungen über die Ausgestaltung der Gemeinsamen Agrarpolitik (GAP) im Jahr 1964, die bei einigen Ländern zur Frustration über die Europapolitik de Gaulles führten.

Schwerpunkt des Buches sind die beiden folgenden Kapitel über die Krise des leeren Stuhls, die Ludlow vor allem als Machtkampf zwischen der Bundesrepublik, den Niederlanden und Italien auf der einen sowie Frankreich auf der anderen Seite interpretiert. Belgien habe die wichtige Rolle übernommen, mäßigend auf die Kontrahenten einzuwirken. Mit dem Luxemburger Kompromiss vom Januar 1966, der die Differenzen über institutionelle Fragen und den Einsatz von Mehrheitsbeschlüssen nicht abschließend klärte, gelang es immerhin, Frankreich wieder an den Ratstisch zurückzuholen. Damit konnten die Arbeiten an der Ausgestaltung der GAP und die Suche nach einer gemeinsamen EWG-Position in der Ken- 
nedy-Runde des GATT wiederaufgenommen werden. Zwischen Mai und Juli 1966 verabschiedete die EWG wichtige Entscheidungen für diese Bereiche.

Als das EWG-Schiff nach dieser stürmischen Phase in ruhiges Gewässer steuerte, kam die englische Beitrittsproblematik erneut auf die Agenda. Die Labour-Regierung unter Premierminister Harold Wilson rang sich dazu durch, einen britischen Beitrittsantrag zu stellen. Ludlow weist zurecht darauf hin, dass, im Gegensatz zum ersten Veto, de Gaulles zweites Veto gegen einen britischen Beitritt vom Dezember 1967 die Arbeiten der EWG fast zum Stillstand brachte. Bereits vorher festgelegte Termine, wie die Vollendung der Zollunion und der Beginn der Endphase des Gemeinsamen Agrarmarkts zum 1.7.1968, konnten noch vollzogen werden. Auch die alltägliche Routinearbeit wurde bewältigt. Aufgrund der Differenzen in der Beitrittsfrage gelang es jedoch nicht, weitere wichtige Integrationsfelder zu erschließen. Vor allem die Niederlande und Italien boykottierten zentrale Vorhaben wie zum Beispiel die Ausarbeitung einer europäischen Technologiepolitik durch die Groupe Maréchal. Erst als sich nach dem Rücktritt de Gaulles im April 1969 eine flexiblere Haltung des neuen französischen Staatspräsidenten Georges Pompidou abzeichnete, kam die europäische Integration wieder in Bewegung. So gelang es den Staats- und Regierungschefs der sechs Länder auf der Haager Gipfelkonferenz im Dezember 1969 sich in der Beitrittsfrage zu einigen und im Gegenzug die Fortführung des für Frankreich vorteilhaften Agrarfinanzierungsmodus zu vereinbaren. Außerdem wurden mit der Währungspolitik und der politischen Zusammenarbeit zwei Politikfelder erschlossen, die für die Zukunft der EWG/EU von zentraler Bedeutung werden sollten. Mit den Beschlüssen der Haager Gipfelkonferenz sieht Ludlow die gaullistische Herausforderung als überwunden an.

Im Zuge seiner Studie kann Ludlow mehrere zentrale Entwicklungen herauspräparieren, die deutlich machen, warum die von Ludlow untersuchte Zeitspanne eine so große Bedeutung für die Geschichte des Integrationsprozesses beanspruchen kann. So veränderte sich unter anderem das Zusammenspiel der Institutionen im Entscheidungsprozess der EWG. Hatte zunächst alles auf eine zunehmend wichtigere Rolle der Kommission hingedeutet, so sieht Ludlow den Ministerrat und den ihm untergeordneten Ausschuss der ständigen Vertreter (COREPER) als institutionellen Gewinner der Phase zwischen 1963 und 1969. In dem untersuchten Zeitraum wurde deutlich, dass keine Regierung gewillt war, auf ihren Einfluss zugunsten der Kommission zu verzichten. Trotz der Bedeutungszunahme des Ministerrats diagnostiziert Ludlow für die Endphase der 1960er Jahre ein „leadership vacuum“ (S.212) für die EWG. De Gaulle polarisierte nur noch und die Bundesrepublik, die sich durch die gaullistische Herausforderung in eine aktivere Rolle gedrängt sah, konnte aus Rücksicht auf den französischen Partner und die eigene Vergangenheit die Führungsrolle nicht kraftvoll genug ausfüllen. Nach dem erfolgreichen Haager Gipfel war im Prinzip klar, dass das ,leadership vacuum“ nur durch regelmäßige Treffen der Staats- und Regierungschefs aufgefangen werden konnte. Von daher lag der Schritt zur Institutionalisierung der Gipfeltreffen, wie er ab 1974 vollzogen wurde, nah. 
Auch im Bereich der Ausgestaltung der vom EWG-Vertrag genannten Politikfelder waren die Jahre zwischen 1963 und 1969 entscheidend. Vor allem wurden die Grundzüge der GAP festgelegt und das Stadium der Zollunion bereits vorzeitig zum 1.7.1968 erreicht. Neue Politikfelder wurden durch den Haager Gipfel erschlossen. Entscheidend für die Zukunft waren auch die Festlegungen im Bereich der Mitgliedschaft. Im Prinzip konnten die Antragsteller Mitglied werden, wenn sie bereit waren, die bisherigen EWG-Regelungen zu übernehmen. Dass sich die EWG anpassen musste, wie noch 1961/63 von Großbritannien gefordert, war jetzt nicht mehr diskutabel.

Insgesamt gelingt es N. Piers Ludlow hervorragend, den Integrationsprozess zwischen 1963 und 1969 zu analysieren und wichtige Entwicklungsprozesse zu verdeutlichen. Dabei versucht Ludlow den europäischen Integrationsprozess auch immer wieder in die Entwicklungen der internationalen Politik der 1960er Jahre einzuordnen. Zudem ruht sein Blick nicht nur auf der Politik Frankreichs, der Bundesrepublik oder Italiens, sondern er streicht an zentralen Stellen auch immer wieder die wichtige Rolle der kleineren Staaten heraus. Das sehr gut lesbare Buch dürfte somit nicht nur zum Standardwerk für die Geschichte der EWG in den 1960er Jahren avancieren, sondern bietet auch den Historikern/Politologen, die sich mit der jüngeren Geschichte der EWG/EU befassen, eine Fülle von Einsichten.

Henning Türk

Universität Duisburg-Essen

\section{QUADRIO CURZIO Alberto - Economisti ed economia. Per un'Italia europea: paradigmi tra il XVIII e il XX secolo (Economists and Economics. In Praise of a European Italy: Paradigms between the XVIII and the XX Centuries), Il Mulino, Bologna, 2007, 387 p.- ISBN 978-88-15-11531-7, 29,00€.}

The volume collects nine essays drafted by Alberto Quadrio Curzio (some in collaboration with other scholars - Claudia Rotondi, Roberto Scazzieri, Mario Talamona) in the twenty years spanning 1986 to 2006.

The essays, mostly historical, economical and institutional in nature and supplied with primary bibliographic references, focus in particular on personalities, mainly economists (C. Beccaria, P. Verri, C. Cattaneo, L. Einaudi, E. Vanoni, P. Saraceno, F. Vito, G. Demaria, G. Fuà), and on the role of distinguished institutes (Istituto Lombardo-Accademia di Scienze e Lettere and Società Italiana degli Economisti) and companies (Edison) that helped promote Italian culture and place it into the European context during the second half of the eighteenth and the twentieth centuries.

In the author's intention, however, the volume is "much more than a collation of essays written in different periods" (p.3). In this respect, it is purposely opened by the introduction, in which Quadrio Curzio outlines and motivates the arrangement of the collection and provides its interpretive key, and insightful conclusive remarks. 
In fact, as pointed out by the author and as emerges from reading the volume, despite being divided into four sections (along chronological and thematic criteria), the essays are closely interrelated by recurrent themes and perspectives. Among these, a focus on the intellectual and operative commitment of those personalities and institutions that promoted fruitful interactions among institutions, market and society in which personal liberty and responsibility "positively" combined - most notably, within this context, Quadrio Curzio identifies an economic-political paradigm defined as 'social liberalism' or 'community liberalism' (p.18) distinguished from both liberalism and socialism -; a pro-European perspective, and the acknowledgment of the role of culture and science in the progress of civilization.

In detail, part one, entitled "Profili di economisti istituzionalisti, liberali ed europeisti: tra XVIII e XIX secolo" (Profiles of institutionalist, liberal and pro-European economists between the eighteenth and the nineteenth centuries), converges on three personalities, Cesare Beccaria (1738-1794), Pietro Verri (1728-1798) and Carlo Cattaneo (1801-1869), considered to be the "makers of the Lombard paradigm" (p.23) - the adjective indicating here the territorial and cultural milieu within the much broader European context in which these versatile scholars carried out their studies in economics, philosophy, law and history. In particular, their economic thought is characterized by open-minded reception of European philosophical and cultural insights and by lively interest in contemporary society; in this respect, they also acknowledge the relevant role played both by institutions, as complementary to individual action in fulfilling needs, and by the civil (economic-commercial, technological, legal) society, as the intermediate body between individuals and the State. They moreover see knowledge as aimed at the definition of guidelines for action for economic governance and the advancement of society as a whole. The volume discusses in particular Verri's and Beccaria's proposal for a monetary reform capable of bringing Italy closer to other European countries on the bases of their economic interdependence (part one, chapter II), and Cattaneo's awareness, as realized in the founding of the "Politecnico" (1839), of the contribution of science and knowledge to economic and social progress (part one, chapter III).

Part two, "Paradigmi economici dell'accademia e dell'industria lombarda: tra XIX e XX secolo" (Economic paradigms of academia and industry in Lombardy between the nineteenth and the twentieth centuries) investigates two different institutional contexts: the Istituto Lombardo - Accademia di Scienze e Lettere and Edison. The Istituto, founded in 1797 on the initiative of Napoleon Bonaparte, began its activity in 1803, and despite political and nominal changes it has unfalteringly maintained, among its key principles and aims, the promotion of scientific knowledge for civil progress to be realized by the continuous dialogue between natural and humanistic sciences and by open-mindedness towards European thought. In this respect, the author examines the scientific production of authoritative Italian economists, emphasizing the role of economic science as a discipline bridging "scientific-technological and legal-philosophical perspectives at 
a cultural level" (p.145). The case of Edison, the energy sector company founded by Giuseppe Colombo (1836-1921) in 1884, is discussed as the living example of the contribution of the application of scientific and technological knowledge to civil progress. The essay retraces the history of the company through its administrators, seen as belonging to the wider group of "engineers-economists and technologists-entrepreneurs" (p.153) that realized economic development opportunities in Northern Italy between the second half of the nineteenth and the beginning of the twentieth centuries, and highlights their unabated commitment to the internationalization of the company and to human resources training as key factors in their entrepreneurial action.

Part three, "Profili di economisti per lo sviluppo istituzional-economico in Europa: il XX secolo" (Profiles of economists committed to institutional and economic development in Europe: the twentieth century) focuses on three economists: Luigi Einaudi (1874-1961), Ezio Vanoni (1903-1956) and Pasquale Saraceno (1903-1991). The essay on Einaudi traces the ripening of his thought upon European federalism during his sixty-year activity (1897-1956). Einaudi envisages European federalism as the resolutive political condition, both to the crisis in the equilibrium of European nations as emerged in the two World Wars and to their social and economic interdependence, and in the Forties and Fifties defines it along a specifically liberal perspective in terms of its political-institutional and economic-institutional structure (bicameral parliament, shared military forces, financial means, market and monetary unification). Einaudi's contribution is essentially theoretical in nature; however, his views significantly influenced those Italian personalities playing an active role in the process of European unification, such as Altiero Spinelli and Alcide De Gasperi. The essay on Vanoni and Saraceno delineates their contribution - the former as engaged in the economic Ministries, the latter as producing models that were to shape Italian economic and industrial policies - to the process of second post-war reconstruction of Italian economy aimed at its re-insertion into the European circuit.

Part four is entitled "Un paradigma associativo tra economisti e tre profili caratterizzanti nella seconda metà del XX secolo" (An associative paradigm among economists, and three key figures in the second half of the twentieth century). It opens by tracing the history of the Società Italiana degli Economisti, from its foundation - in 1950, during an economists' meeting in Turin --, to its statutory changes (in 1984 and 1998) and its most recent evolution, and underlines the significant extent to which the Associazione has played a key role in promoting and supporting the progress of economic science in Italy. The personalities and thought of three of its key members, that is the economists Francesco Vito (1902-1968), Giovanni Demaria (1899-1998), Giorgio Fuà (1919-2000), are then illustrated in the final essay.

In the conclusion Quadrio Curzio returns to the findings presented in the volume in the light of a reflection on their significance today, and outlines the main features of his 'social liberalism', or 'community liberalism', paradigm as anticipated in the Introduction and discussed, among others, also in: Noi, 
l'economia e l'Europa, Bologna, Il Mulino, 1996; Sussidiarietà e sviluppo. Paradigmi per l'Europa e per l'Italia, Milano, Vita e Pensiero, 2002.

The volume by Quadrio Curzio represents thus a crucial contribution to the definition of the identity of Italy within the European context and a fruitful source of stimulating inspiration for future theoretical and operative paths.

Ilaria Pasotti

University of Florence-Università degli Studi di Firenze

LUDLOW Piers (ed.) - European Integration and the Cold War. Ostpolitik -Westpolitik, 1965-1973, (Cold War History Series 17), Routledge, London, 2007, 194 p. - ISBN 978-0-415-42109-6 (hb), 95,29 €.

The historiography of European integration largely developed out of the historiography of the Cold War. Since its beginnings in the late 1970s and early 1980s, however, it has outgrown to become an independent research field in contemporary (European) history in its own right - a research field, moreover, whose subject matter, the present-day European Union (EU), is alive and kicking compared to the post-war Cold War which ended in this form around 1990. As Piers Ludlow claims with some justification in the introduction to this book, however, "the history of each has tended to be studied and told with next to no reference to the other" (p.1). Some scholars like Wilfried Loth and now Ludlow himself continue to straddle the growing divide between the two research fields, but most concentrate on one or the other, with their different networks, conferences and publication outlets. Yet, as Ludlow argues in the conclusion, the Cold War and European integration "did not take place in hermetically sealed containers entirely prevented from contact with one another" (174). This edited book sets out to redress this apparently sorry state of affairs and to reconnect Cold War and European integration historiography by focussing on the period from the "Empty Chair" crisis in 1965 to the first enlargement of the European Communities (EC) at the beginning of 1973. In part this is a pragmatic choice as new sources from government archives have become available. It is also an interesting transition period, however, with new internal and external policy challenges and the partial replacement of the France of Charles de Gaulle with the Britain of Harold Wilson (from 1974) as the EC's (most) "awkward partner" (Stephen George).

The nine chapters in this book address different issues covering the entire time-span or only, in one case, seven months. In the first chapter, Georges-Henri Soutou contrasts the approaches of de Gaulle and his successor as French president, Georges Pompidou, towards the Cold War and integration issues. In a diplomatic historical perspective on national foreign policy-making, Soutou re-states in a variation of one of many European "special path" theses about national histories that "as usual, the French are a special case". Somewhat surprisingly for scholars of the history of the present-day EU, this was not least the case because both presidents were "against European integration" (p.11). Why 
then, one might be tempted to ask, did de Gaulle fight so vigorously for the very supranational Common Agricultural Policy and Pompidou support the plan for monetary integration?

In the second chapter, Garret Martin studies de Gaulle's second veto against British membership, bizarrely starting his analysis in September 1967 despite clear indications that the French president never changed his mind on the issue since the first veto, something clearly understood by the British government. Like several other authors in this book, Martin uses traditional realist language endowing countries with emotions such as when he argues, for example, that "France" "distrusted Britain" (p.44). In the third chapter, Wilfried Loth - partially based on sources from the French Archives nationales collected for him by Claudia Hiepel discusses the relationship between Pompidou and the new social democratic German chancellor Willy Brandt from 1969 to 1974. In the subsequent chapter, Andreas Wilkens revisits the links between Brandt's Ostpolitik and EC politics, which perhaps provided the most important connection between the Cold War and European integration as it also induced Pompidou to support British accession.

The following two chapters by Helen Parr and James Ellison discuss from a British perspective the second British application for membership and Anglo-American relations over the issue. Parr evidently likes the Labour prime minister very much, arguing that "Wilson sowed the seeds of a British vision of the European Community that flourished only later" (p.101), although what this "vision" was precisely, remains unclear. Indeed, subsequent British governments have usually claimed that the "vision thing" was not for the allegedly pragmatic British, but characteristic of mad continental European federalists. Indeed, what appears to be emerging in outlines in these more recent studies of British policy, which are as Anglo-centric as ever, is a version of EU history somewhat reminiscent of Niall Ferguson's perspective on British Empire which once more appears to have spread a glorious civilisation at a very small cost - in this case at least only of repetitive referendum, budget, negotiation and media crises in the EU, not hundreds of thousands of dead indigenous people in newly subjugated colonies. At the same time, Ellison's competent exposition of the boring topic of Anglo-American relations over the second British EC application inadvertently demonstrates the disjuncture between the Cold War and European integration, as US support neither influenced the second British application nor even any more de Gaulle's opposition to it.

In his mini chapter of only nine pages, Jan van der Harst reiterates how subsequent Dutch governments were torn between their supranational institutional and policy preferences for the EC and their traditionally Atlantic foreign policy outlook on NATO, relations with the US and British EC accession. Ludlow then analyses how the European Commission related to the Cold War. In what he calls its own "bureaucratic logic" (p.144), the Commission's policy concerns were primarily of a domestic EC nature and not related to conflicts in NATO, for example. Indeed, as Ludlow rightly claims (p.147), the relative stability of NATO and the US role in providing security for Western Europe in the Cold War made it 
so much easier for the Community to concentrate on the "low politics" of integration in fields like agriculture and competition policy, for example. At the same time, the early 1970 s saw only a very modest collective attempt with the formation of the European Political Cooperation to create some limited foreign policy autonomy from the western superpower. Finally, Jussi Hanhimäki, in the ninth chapter, argues that two crucial conditions for close Atlantic cooperation remained largely constant in this period, that is the convergence of basic economic interests despite petty trade wars and the importance of NATO for Western Europe as a security shield. Hanhimäki also shows that "Europe" was not in fact a central policy concern for US administrations in this period (p.153) which had to deal with other challenges such as the war in Vietnam and the collapse of the Bretton Woods system. The US did succeed, however, in avoiding a breakdown of Western unity or the creation of a much more autonomous European foreign policy - although arguably, neither one nor the other required much US activism but in fact reflected the preferences of most, if not all, NATO partners.

Many of the empirical insights in this chapter are accessible in more elaborate form in recently published books, for example by Parr on Wilson's European policy or Ellison on Anglo-American relations over "Europe", or they have circulated for a very long time as in Wilkens' many publications in French and German on exactly the same topic as his book chapter, or they form part of larger projects like Hiepel's Habilitation on the European concepts and policies of Brandt and Pompidou in the case of Loth's chapter. Nonetheless, it can of course be useful to bring them together in an edited volume, although it is plainly annoying that such an expensive book should contain so many printing errors (especially missing French special signs with a loss of their accompanying letters), for example eleven on page 8 of the introduction and an incredible 23 on page 65 of Loth's chapter. Yet, the chapters do not attempt to address a common set of questions, they make no cross-references, and they mainly appear to be united by a traditional diplomatic historical approach to understanding national European policy and inter-state relations. The central problem is of course that this may be an adequate framework for analysing "high politics" security and military issues, although it is a limited approach even there, but that it is wholly inadequate for the historical reconstruction of EU politics in historical perspective, something that requires a sophisticated understanding of transnational and supranational dimensions of this emerging polity.

As Ludlow himself argues, links between the Cold War and European integration were largely limited to strategic issues which had to be addressed by heads of government and foreign ministries in particular. Conflicts within the EC and in transatlantic relations became more virulent over time in all areas in which the EC slowly, but surely acquired external competences and increased its cohesion as an international actor in fields like trade and development policy and foreign policy cooperation, for example during the Helsinki process in the 1970s. If historians of the present-day EU want to invest resources in the study of connections between the Cold War and European integration they should perhaps 
focus on these policy areas and related issues from a multilateral perspective, not petty questions such as the repetitive begging of British prime ministers to be allowed to join the EC in the vain hope that this in itself would deal with all domestic structural problems of a former Empire in temporary (as it eventually turned out), not terminal decline.

Wolfram Kaiser

University of Portsmouth

\section{Rainer EISING, Beate KOHLER-KOCH (Hg.), Interessenpolitik in Europa, Baden-Baden, Nomos, 2005, 389 S. - ISBN 978-3-8329-0779-2 - 69,00 €.}

This important book addresses the role of (in the broadest sense: economic) interest associations in the process of European integration. It collects theoretically informed empirical studies offering precise and deep insights into the structural preconditions and the ways and means of interest articulation, representation and achievement on the European level. The methodological spectrum ranges from qualitative and quantitative approaches to case studies and comparisons, the framing of models and "thick descriptions". Most of the contributions prefer a multi-level perspective, and the focus lies on North and West European countries. The editors claim with full justification to present an overview of the German-speaking political science research on problems of EU interest politics.

The first part of the book deals with theoretical approaches and conceptions. One of the most interesting articles explores changes concerning the logic of influence within the European Union: Taking the German Farmer's Association and the Federation of German Banks as examples, Dieter Wolf stresses the possibility to guarantee or at least affect their member's compliance with EU-regulations. The other articles of this part discuss lobbying activities directed to the European Parliament and the role of bureaucrats in the process of European integration.

The authors of the second part of the book examine various policy areas and protagonists, for example the financial market integration, the "Association for the Monetary Union of Europe" or the growing importance of commercial consultancies within the EU. The third part is devoted to the "Europeanization" of interest representation and mediation. Topics range from the formulation of national bargaining positions as a preparation for negotiations on the EU level to collective bargaining between the "social partners" concerning industrial law and the strategies of adaptation of economic associations to external challenges. As far as the last topic is concerned, Achim Lang and Jürgen R. Grote show that changing "political environments", especially a growing "Europeanization", cause adjustment efforts on the side of the associations. And Rainer Eising proves that the EU increases the power of associations already distinctive and established on a national level.

Although some of the authors have chosen a rather abstract level of examination, historians of European integration may gain in many ways from the 
corresponding research of political scientists. Focussing and discussing problems of "democracy", "efficiency", or "interest representation" with regard to national or European interest organisations is without doubt important for them, too.

Prof. Dr. Werner Bührer Technische Universität München 


\title{
Abstracts - Résumés - Zusammenfassungen
}

\author{
Wilfried Loth \\ Explaining European Integration: The Contribution from Historians
}

\begin{abstract}
The debate on the present results of research in the history of European integration leads to a model of "four driving forces" behind European integration: the maintenance of peace among sovereign states in Europe, the resolving of the German question, the preservation of economic productivity, and the self-assertion in the face of new world powers. From the development of these driving forces there may be deduced both the timing of specific integration initiatives as well as the decision for specific types of integration. The European Economic Community appears primarily as a political construction which only gradually gained economic attractiveness. Since the end of the Cold War the political impulses have become more important once again. At the same time, one may notice tendencies towards social integration as well as towards an increased consensus of the Europeans regarding their conceptions of political values. The central question of future integration research will concern the relationship of democratic legitimacy and efficiency in political decision-making. In search of answers, historians won't fail to point out that things being in a state of flux, the possible will be not only what used to be possible.
\end{abstract}

\section{Les contributions de l'historiographie à l'interprétation de l'intégration européenne}

La discussion des résultats actuels de la recherche historiographique relative à l'intégration européenne débouche sur le modèle des «quatre moteurs» de l'intégration européenne: le maintien de la paix entre Etats souverains en Europe, la solution de la question allemande, la préservation de la productivité économique et la volonté de s'affirmer face aux nouvelles puissances mondiales. A partir du développement de ces moteurs, on peut déduire à la fois le moment où sont intervenues certaines étapes du processus d'intégration et les formes d'intégration finalement retenues. La Communauté économique européenne semble ainsi représenter en première ligne une construction politique dont l'attractivité économique se déploie seulement par après. Depuis la fin de la Guerre Froide, les impulsions politiques gagnent en importance. Simultanément des tendances à l'intégration sociale et au renforcement du consensus autour des valeurs politiques des Européens se manifestent. La question centrale de la recherche en matière d'intégration concernera à l'avenir la relation entre la légitimité démocratique et l'efficacité des décisions politiques. Lors de la quête pour trouver des réponses les historiens ne manqueront pas de souligner que les choses bougent. Partant il faudra envisager qu'à l'avenir il y aura d'autres choix possibles que ceux qui ont depuis toujours été possibles.

\section{Beiträge der Geschichtswissenschaft zur Deutung der europäischen Integration}

Die Diskussion der Ergebnisse bisheriger Forschungen zur Geschichte der europäischen Integration führt zu einem Modell von ,,vier Antriebskräften“ europäischer Integration: Friedenssicherung unter souveränen Nationalstaaten in Europa, Lösung der Deutschen Frage, Sicherung wirtschaftlicher Produktivität, Selbstbehauptung gegenüber neuen Weltmächten. Aus der Entwicklung dieser Antriebskräfte lassen sich sowohl der Zeitpunkt für bestimmte Integrationsschritte ableiten als auch die Entscheidung für bestimmte Arten der Integration. Die Europäische Wirtschaftsgemeinschaft erscheint danach in erster Linie eine politische 